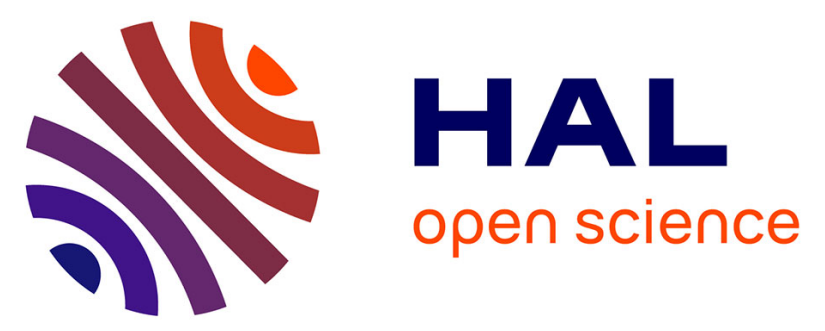

\title{
Numerical investigation of generalized Graetz problem in circular tube with a mass transfer coupling between the solid and the liquid
}

\author{
Franck Pigeonneau, B Jaffrennou, A Letailleur, K Limouzin
}

\section{To cite this version:}

Franck Pigeonneau, B Jaffrennou, A Letailleur, K Limouzin. Numerical investigation of generalized Graetz problem in circular tube with a mass transfer coupling between the solid and the liquid. International Journal of Heat and Mass Transfer, 2016, 10.1016/j.ijheatmasstransfer.2016.01.040 . hal-01263901

\section{HAL Id: hal-01263901 \\ https://hal.science/hal-01263901}

Submitted on 28 Jan 2016

HAL is a multi-disciplinary open access archive for the deposit and dissemination of scientific research documents, whether they are published or not. The documents may come from teaching and research institutions in France or abroad, or from public or private research centers.
L'archive ouverte pluridisciplinaire HAL, est destinée au dépôt et à la diffusion de documents scientifiques de niveau recherche, publiés ou non, émanant des établissements d'enseignement et de recherche français ou étrangers, des laboratoires publics ou privés. 


\title{
Numerical investigation of generalized Graetz problem in circular tube with a mass transfer coupling between the solid and the liquid
}

\author{
F. Pigeonneau ${ }^{\mathrm{a}, *}$, B. Jaffrennou ${ }^{\mathrm{b}}$, A. Letailleur ${ }^{\mathrm{b}}$, K. Limouzin ${ }^{\mathrm{c}}$ \\ ${ }^{a}$ Surface du Verre et Interfaces, UMR 125 CNRS/Saint-Gobain, 39 quai Lucien Lefranc - BP 135, \\ 93303 Aubervilliers cedex, France \\ ${ }^{b}$ Saint-Gobain Recherche, 39 quai Lucien Lefranc - BP 135, 93303 Aubervilliers cedex, France \\ ${ }^{c}$ Saint-Gobain Performance Plastics, Z.I. de Chesnes, 5 rue du Dauphiné, 38297 Saint-Quentin \\ Fallavier, France
}

\begin{abstract}
The unsteady diffusion equation in a tube and the advection/diffusion equation in laminar flow in a liquid of a solute are theoretically established. The most important parameters are the migration strength $\alpha$ and the so-called Graetz number Gr. The former dimensionless number is the product of the partition coefficient of the solute at the solid/liquid interface, the ratio of the tube thickness to the interior radius and the ratio of diffusion coefficients of the solute in the liquid and in the solid. The Graetz number Gr is the ratio of the diffusion time scale in the liquid based on the interior radius of the pipe to the advection time over the tube length. The problem is applied to the plasticizer migration from a polyvinyl chloride material to a liquid with the safety food applications in mind. The migration of the plasticizer is solved numerically for various tube sizes, flow conditions and partition coefficients of the plasticizer in the liquid. For comparison, the numerical results obtained in static condition are also provided.

The average plasticizer concentration in the liquid behaves following two main regimes as a function of the migration strength. When $\alpha$ is much smaller than one, the average plasticizer concentration in the liquid is an algebraic function of the axial coordinate, $z$, at the power two third and increases linearly with time. Conversely, when $\alpha$ is much larger than one, the average plasticizer concentration is linear as a function of axial distance and increases as a function of the square root of time. Moreover, the concentration is much smaller of few orders of magnitude in dynamic condition than in static working showing that in the context of food contamination the dynamic approach is more appropriate and relevant for demonstration of compliance with the safety authorities requirements.
\end{abstract}

Keywords: Graetz problem, migration, plasticizer, food safety, contamination

\footnotetext{
*Corresponding author: Tel. +33 (1) 483959 99, Fax +33 (1) 48395562.

Email addresses: franck.pigeonneau@saint-gobain.com (F. Pigeonneau), boris.jaffrennou@saint-gobain.com (B. Jaffrennou), alban.letailleur@saint-gobain.com (A. Letailleur), krystel.limouzin@saint-gobain.com (K. Limouzin)
} 


\section{Introduction}

Heat transfer between a fluid flowing through a solid tube is commonly used to design heat mass exchanger devices, see the recent book of Zhang [1]. Since the first contributions of Graetz [2,3], the heat transfer between a solid and a liquid has been extensively studied and summarized in the book of Shah and London [4]. According to $[5,6]$, the analogy between heat and mass transfer in the limit of mass diffusion of a solute in weak concentration in a solvent can be invoked meaning that the same physics can be applied to migration issues.

The last situation occurs in the food delivery through tubes made from thermoplastic matrices involved in milk producing and transforming, vending machines among others. The contact between food and its container leads to a release of additives in the food. According to safety requirements, articles or materials in contact with food have to be in compliance dictated by the food safety authorities. Two quantities have to be mainly determined to prove the compliance: the former is the overall migration corresponding to the total amount of material migrating in the food per unit of surface of the container in contact with food, measured in $\mathrm{mg} / \mathrm{dm}^{2}$. The latter is the specific migration corresponding to the migration of particular substances by mass unit of food in the container. The European Union (EU) regulation 10/2011 [7] sets the Overall Migration Limit (OML) equal to $10 \mathrm{mg} / \mathrm{dm}^{2}$. For substances without Specific Migration Limit (SML), a generic SML equal to $60 \mathrm{mg} / \mathrm{kg}$ shall apply. However, this value can be reduced for certain substances like for polydimethylsiloxane, for which the SML is equal to $6 \mathrm{mg} / \mathrm{kg}$, for instance.

The compliance of a plastic material should be measured using food simulants under specified test conditions. The specification of EU regulation has been based on static conditions, for which food simulant is in contact with the container during a certain time. Nevertheless, for deliveries through hoses, liquid food is in motion. Consequently, the mass transfer with solid/liquid interfaces exhibits different behaviors in comparison with the static situation. Additives are transported with the food limiting the accumulation. Mass transfer involves mass diffusion in the tube and advection of the liquid food. Consequently, the migration process in dynamic conditions has to be studied in more details, which is the aim of this work. The important questions which arise are the following: What are the main differences between migration without or with motion? What will be the consequences in terms of safety requirements? In order to reply to those questions, this article is devoted to the numerical modelling of the dynamic and static conjugated migration problems. It is noteworthy to say that the problem addressed here is general and can be also applied to the heating or the cooling of a tube.

The conjugated problem has been addressed for the first time for heat transfer applications by Mori et al. [8] and latter by Faghri and Sparrow [9] in the situation of high conduction in the solid meaning that only the steady state regime has been studied. A special attention to axial conduction has been investigated in $[10,11,12]$ but once again in steady state regime both in the liquid and in the solid. More recently, Zhang et al. [13] achieved a numerical work of the conjugated problem by solving the Navier-Stokes equations and the two energy equations. A parametric study was carried out on the ratio of thermal diffusivity and on the ratio of the thickness of solid tube to the interior diameter of the pipe.

In the framework of safety food requirements, numerical simulations are generally 
used with a simple 1-D geometry. The migration model is generally based on the development provided by Crank [14] to determine the migration of a substance well stirred in the solution (food simulant). The process is only limited by diffusion in the PVC sheet since the solution is seen as a perfect diffuser. Begley et al. [15] summarized the main steps to build up and validate a migration estimation model. They compiled a large amount of data in order to determine the "worst-case" migration level. The method requires the knowledge of diffusion coefficients of additives in the packaging material and of the partition coefficients which define the chemical equilibrium at the food/container interface. Vitrac et al. [16] described the migration model based on the local diffusion process requiring the mass transfer coefficient in the food simulant depending on fluid motion of the simulant. They identified diffusion, partition, and mass transfer coefficients by an optimized calculation. Brandsch et al. [17] summarized how the mathematical modeling can be used to evaluate the migration limits.

The numerical simulation of migration process in a cylindrical tube has been seldom investigated. Let us quote nevertheless, works achieved in the framework of water distribution for which the diffusion of antioxidant from hoses to hot-water has been investigated by Smith et al. [18] and more recently by Dear and Mason [19]. The diffusion of chlorine from water to the tube is also taken into account and reacts with the antioxidant substance following an irreversible reaction. Nevertheless, the water motion is not addressed in previous works. Mittelman et al. [20] studied the same problem for which an exact solution was proposed when chemical reactions are neglected. Otherwise, a numerical simulation was established with a finite difference technique.

The dynamic condition of migration of plasticizer requires a special attention which is the main purpose of the present work. Indeed, apart from the flowing behavior, the migration process is studied for hoses for which the dilution can be strongly limited for small tube. Moreover, the diffusion process exhibits particular behaviors like for instance the tube curvature. In order to study the migration, a theoretical model is developed and solved numerically to describe the migration in dynamic conditions. Since the aim of this present study is to compare the migration in static and dynamic conditions, the conjugated problem of diffusion between two media at rest is also presented and solved numerically. A special attention will be done on the amount of additive migrating in the liquid at a function of time.

In the following, the problem statement of the dynamic migration is described in the section 2 in which a dimensionless formulation is built pointing out the relevant dimensionless numbers. Section 3 is devoted to the presentation of results and discussion in which the effects of the geometry, flow rate and plasticizer solubility are addressed before to conclude in $\S 4$. Appendix A presents the conjugated static migration problem. Details of the numerical methods both for the dynamic and static problems are provided in supplementary material of this article.

\section{Problem statement of the dynamic conjugated migration}

Although the migration process is addressed both in static and dynamic conditions, only the conjugated problem of mass diffusion in dynamic condition is presented below. Since the static problem has been considered elsewhere, the presentation in this last situation is reported in Appendix A. 
Only one substance is considered in the problem. In the solid tube, the mass concentration of the plasticizer is written $C_{p}$ and $C_{f}$ in the liquid. The diffusion coefficients of the plasticizer are denoted $D_{p}$ and $D_{f}$ in the solid and in the liquid respectively. The plasticizer concentration in the pipe is in large amount in order to increase the flexibility.

As it is well known in materials science, the diffusion of plasticizer in solid material depends upon the plasticizer concentration [21]. A decrease of plasticizer concentration changes the temperature of the glass transition of the PVC material, decreasing drastically the diffusion coefficient [22]. It is worthy to note that all over this work, the diffusion coefficients are assumed in first approximation constant over the time of migration process. This assumption means that the mass transfer will be limited to the first times in order to avoid a large decrease of plasticizer in the PVC material. In fact, the time scale of diffusion in the PVC material is inversely proportional to the diffusion coefficient. The typical value of the time scale of diffusion for a tube of one millimeter in thickness is larger than 2700 hours by taking a diffusion coefficient $D_{p}$ equal to $10^{-13}$ $\mathrm{m}^{2} / \mathrm{s}$ which is a typical value for a plasticizer in flexible PVC tube [23]. The time is very high in comparison with the typical times of migration experiments which do not exceed few tens of minutes meaning that the decrease of plasticizer concentration stays low. Note also that by assuming a constant value for the diffusion coefficient, the migration process is treated in the worst situation, since when the diffusion coefficient decreases as a function of time, a skin layer acting as a functional barrier against migration is created. Moreover, the aim here is to compare with the static situation for which the same assumption is used. The change of diffusion coefficient as a function of the plasticizer concentration should not modify the main conclusions of the present investigation.

In the liquid, the diffusion coefficient is determined assuming that the additive concentration in the liquid is sufficiently small in order to apply the Wilke and Chang's correlation [24]. A numerical value will be provided at the beginning of section 3 .

Figure 1 summarizes the problem statement where typical profiles of $C_{p}$ and $C_{f}$ are sketched. The tube length is $L$, and interior and exterior radii are $r_{i}$ and $r_{e}$ respectively. The liquid flows with a volumetric flow rate equal to $Q$ and has a constant density, $\rho$ and dynamic viscosity, $\mu$. In the following, the flow regime is assumed laminar meaning that the typical Reynolds number is less than 2100 [5]. In this regime, the velocity profile is well known and given by the Hagen-Poiseuille solution [25]:

$$
U=\frac{2 Q}{\pi r_{i}^{2}}\left[1-\left(\frac{r}{r_{i}}\right)^{2}\right] .
$$

To describe the migration process, mass conservation has to be written both in the solid and in the liquid. In the solid, the unsteady diffusion equation is

$$
\frac{\partial C_{p}}{\partial t}=D_{p}\left[\frac{1}{r} \frac{\partial}{\partial r}\left(r \frac{\partial C_{p}}{\partial r}\right)+\frac{\partial^{2} C_{p}}{\partial z^{2}}\right],
$$

in which $t$ is the time. The cylindrical coordinate system is employed for which $r$ is the radial and $z$ the axial coordinates as depicted in Figure 1. This last equation is written in space $\left.(r, z) \in] r_{i}, r_{e}[\times] 0, L\right]$. In the liquid, the mass conservation takes the following 


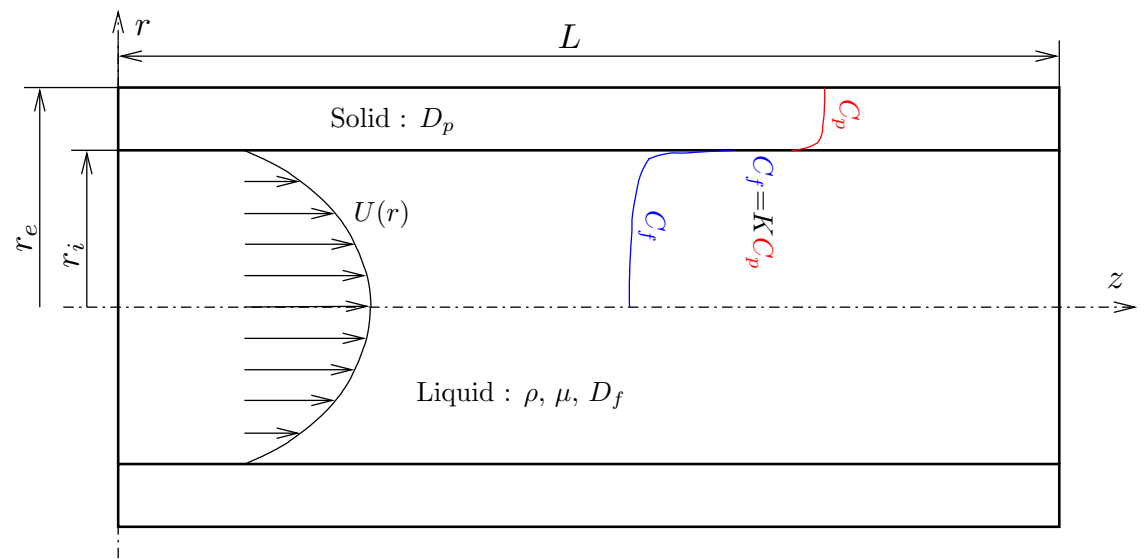

Figure 1: Geometry and description of the migration physics in a tube of length $L$, interior radius $r_{i}$ and exterior radius $r_{e}$.

form

$$
\frac{\partial C_{f}}{\partial t}+U(r) \frac{\partial C_{f}}{\partial z}=D_{f}\left[\frac{1}{r} \frac{\partial}{\partial r}\left(r \frac{\partial C_{f}}{\partial r}\right)+\frac{\partial^{2} C_{f}}{\partial z^{2}}\right]
$$

written when $(r, z) \in\left[0, r_{i}[\times] 0, L\right]$.

These two last equations have to be completed by boundary and initial conditions. First, the atmosphere outside the tube is assumed to be without plasticizer all the time. Consequently, the mass flux from the tube is considered to be equal to zero at any time:

$$
\frac{\partial C_{p}}{\partial r}=0, \text { when } r=r_{e} .
$$

At the interface between the solid tube and the liquid, two conditions have to be written: one arises from the mass conservation and the second from the local thermodynamic equilibrium. The former is obtained by the mass balance at the solid/liquid interface which in absence of relative normal velocity is reduced to the balance of molecular mass fluxes between the two matters. The latter comes from the equilibrium of chemical potentials in the liquid and in the solid [26]. This condition is equivalent to the Henry's law [27]. The respective relations are

$$
\begin{aligned}
D_{p} \frac{\partial C_{p}\left(r_{i}, z, t\right)}{\partial r} & =D_{f} \frac{\partial C_{f}\left(r_{i}, z, t\right)}{\partial r} \\
C_{f}\left(r_{i}, z, t\right) & =K C_{p}\left(r_{i}, z, t\right) .
\end{aligned}
$$

The quantity $K$ is the partition coefficient which can be determined from the Flory theory [28] as achieved by Vitrac and Gillet in [26]. Remark here that the partition coefficient is defined as the ratio of the plasticizer concentration in the liquid to its concentration in the solid, which is the contrary of the definition given in [17]. Moreover, the solution 
is symmetric with respect the $z$-axis meaning that $C_{f}(0, z, t)$ verifies the relation:

$$
\frac{\partial C_{f}(0, z, t)}{\partial r}=0 .
$$

At the entrance of the tube, the concentration of plasticizer is equal to zero in the fluid:

$$
C_{f}(r, z=0, t)=0 .
$$

Initially, the concentration of plasticizer in the tube is uniform:

$$
\left.\left.C_{p}(r, z, 0)=C_{p 0}, \forall r \in\right] r_{i}, r_{e}\right] \text { and } \forall z \in[0, L],
$$

and equal to zero in the liquid:

$$
\left.\left.C_{f}(r, z, 0)=0, \forall r \in\right] 0, r_{i}\right] \text { and } \forall z \in[0, L] .
$$

\subsection{Dimensionless equations}

The normalization of the problem is now proceeded. First, due to the initial conditions and the thermodynamic relation, $C_{p}$ and $C_{f}$ are respectively normalized as follows

$$
\bar{C}_{p}=\frac{C_{p}}{C_{p 0}}, \bar{C}_{f}=\frac{C_{f}}{K C_{p 0}} .
$$

The radial coordinate is reduced following two dimensionless forms. First, since the diffusion process in the solid material occurs in an annular geometry, the relevant geometry scale is $\Delta r=r_{e}-r_{i}$. Consequently, $r$ is written for the annular region as follows

$$
r=r_{i}+\Delta r \zeta
$$

for which $\zeta \in[0,1]$. Second, for the mass balance in the fluid, the radial coordinate is obviously reduced like

$$
\bar{r}=\frac{r}{r_{i}}=1+\Delta \bar{r} \zeta,
$$

with

$$
\Delta \bar{r}=\frac{\Delta r}{r_{i}} .
$$

The axial coordinate and the time are normalised by

$$
\bar{z}=\frac{z}{L}, \bar{t}=\frac{t}{t_{0}},
$$

in which the time scale, $t_{0}$, will be specified below. Finally, the velocity field $U$ is normalized by the average velocity written as follows

$$
\bar{U}=\frac{U \pi r_{i}^{2}}{Q} .
$$


The equations for the concentrations in the solid and in the fluid become

$$
\begin{gathered}
\frac{\Delta r^{2}}{D_{p} t_{0}} \frac{\partial \bar{C}_{p}}{\partial \bar{t}}=\frac{1}{1+\Delta \bar{r} \zeta} \frac{\partial}{\partial \zeta}\left[(1+\Delta \bar{r} \zeta) \frac{\partial \bar{C}_{p}}{\partial \zeta}\right]+\left(\frac{\Delta r}{L}\right)^{2} \frac{\partial^{2} \bar{C}_{p}}{\partial \bar{z}^{2}} \\
\frac{\pi r_{i}^{2} L}{Q t_{0}} \frac{\partial \bar{C}_{f}}{\partial \bar{t}}+\bar{U}(\bar{r}) \frac{\partial \bar{C}_{f}}{\partial \bar{z}}=\frac{1}{\operatorname{Gr}}\left[\frac{1}{\bar{r}} \frac{\partial}{\partial \bar{r}}\left(\bar{r} \frac{\partial \bar{C}_{f}}{\partial \bar{r}}\right)+\left(\frac{r_{i}}{L}\right)^{2} \frac{\partial^{2} \bar{C}_{f}}{\partial \bar{z}^{2}}\right],
\end{gathered}
$$

for which

$$
\mathrm{Gr}=\frac{Q}{D_{f} \pi L},
$$

is the Graetz number measuring the ratio of the diffusion time scale over the radius $r_{i}$ to the advection time scale over the tube length $L$.

In practical cases, the tube length is always much larger than the radial dimensions meaning that ratios $(\Delta r / L)^{2}$ and $\left(r_{i} / L\right)^{2}$ are assumed much smaller than one. In this limit, the derivatives respect to the longitudinal coordinates can be neglected. According to equations (17) and (18), the time scale $t_{0}$ must be taken as

$$
t_{0}=\max \left(\frac{\Delta r^{2}}{D_{p}}, \frac{\pi r_{i}^{2} L}{Q}\right) .
$$

Since as already indicated at the beginning of this section, the typical time scale of diffusion of the liquid is very high (larger than one thousand of hours) which is always much larger than the time scale of advection. Consequently, the time scale $t_{0}$ is taken equal to $\Delta r^{2} / D_{p}$. From this scaling analysis, one observes that the time derivative of $\bar{C}_{f}$ in Eq. (18) is multiplied by a factor equal to $D_{p} /\left(\operatorname{Gr} \Delta \bar{r}^{2} D_{f}\right)$ which can be seen as a Strouhal number or an homochronous number according to Luna et al. [29] who studied the conjugated heat transfer in circular ducts for a non-Newtonian fluids. Since in the framework of our applications the diffusion coefficient in the solid is three order of magnitude smaller than the diffusion coefficient in the liquid and that the Graetz number is usually larger than one, $D_{p} /\left(\operatorname{Gr} \Delta \bar{r}^{2} D_{f}\right)$ is expected to be much smaller than one. This means that the time derivative of $\bar{C}_{f}$ can be neglected. This assumption provides a model so-named Quasi-Steady State Approximation (QSSA). In this limit, the mass transfer coefficient does not depend on time. The unsteady problem has been studied in the framework of mass transfer around a spherical droplet by Juncu and Mihail [30]. They show that the steady mass transfer coefficient is reached over a typical time scale around $r_{i}^{2} / D_{f}$ which is very smaller than $\Delta r^{2} / D_{p}$ meaning the QSSA model is relevant in our case.

Consequently, two problems have to be addressed. In the following, in order to lighten notations, we drop the bar over dimensionless variables. The first describing the diffusion/advection problem in the liquid obeys the parabolic partial differential equation:

$$
U(r) \frac{\partial C_{f}}{\partial z}=\frac{1}{\operatorname{Gr} r} \frac{\partial}{\partial r}\left(r \frac{\partial C_{f}}{\partial r}\right),
$$

in which $\mathrm{Gr}$ is the Graetz number given by Eq. (19).

Here, $C_{f}$ is a function of $r, z$ and $t$ is only a parameter resulting from the coupling with the second problem. In order to separate independent variables to parameters, the 
semicolon is used as follows $C_{f}(r, z ; t)$.

The Graetz problem has been previously solved in various contributions summarized for example in $[31,6]$. According to these previous works and using the analogy between heat and mass transfer, the mass flux of $C_{f}$ at the solid/liquid interface is given by

$$
\frac{\partial C_{f}}{\partial r}(1, z ; t)=\operatorname{Sh}(\mathrm{Gr}, z)\left[C_{f}(1, z ; t)-\left\langle C_{f}\right\rangle(z ; t)\right],
$$

in which $\operatorname{Sh}(\mathrm{Gr}, z)$ is the Sherwood number which is a function of the Graetz number and $z$ given by [31]

$$
\begin{aligned}
& \mathrm{Sh}(\mathrm{Gr}, z)=1.8285+0.2323\left(\frac{\mathrm{Gr}}{z}\right)^{0.488} \exp \left(-14.3 \frac{z}{\mathrm{Gr}}\right) \text { if } \frac{\mathrm{Gr}}{z}<250, \\
& \mathrm{Sh}(\mathrm{Gr}, z)=0.8548\left(\frac{\mathrm{Gr}}{z}\right)^{1 / 3}-0.35, \text { if } \frac{\mathrm{Gr}}{z} \geq 250 .
\end{aligned}
$$

Remark that eq. (24) is singular in $z=0$ meaning that the mass transfer is very efficient at the entrance of the tube.

In equation $(22),\left\langle C_{f}\right\rangle(z ; t)$ is the average concentration of the additive weighted by the flow rate over the radial coordinate as follows

$$
\left\langle C_{f}\right\rangle(z ; t)=\frac{\int_{0}^{1} r U(r) C_{f}(r, z ; t) d r}{\int_{0}^{1} r U(r) d r} .
$$

By integration of (21) over the radial coordinate, $\left\langle C_{f}\right\rangle(z ; t)$ obeys to the following equation

$$
\frac{d\left\langle C_{f}\right\rangle(z ; t)}{d z}=\frac{2 \mathrm{Sh}(\mathrm{Gr}, z)}{\mathrm{Gr}}\left[C_{f}(1, z ; t)-\left\langle C_{f}\right\rangle(z ; t)\right] .
$$

Since in the last equation, $C_{f}(1, z ; t)$ is equal to the concentration of plasticizer in the solid, equation (26) achieves the coupling between the mass fluxes in the liquid and in the solid. At the entrance of the tube, the plasticizer concentration in the fluid is equal to zero giving the following boundary condition

$$
\left\langle C_{f}\right\rangle(0 ; t)=0 .
$$

The mass concentration, $C_{p}$, is the purpose of the second problem. It is a function of $(\zeta, t ; z)$ for which $z$ is only seen as a parameter ${ }^{1}$, which verifies the partial differential equation given in dimensionless form by

$$
\frac{\partial C_{p}}{\partial t}=\frac{1}{1+\Delta r \zeta} \frac{\partial}{\partial \zeta}\left[(1+\Delta r \zeta) \frac{\partial C_{p}}{\partial \zeta}\right]
$$

\footnotetext{
${ }^{1}$ The semicolon is once again used to separate independent variables $\zeta, t$ to the parameter $z$.
} 
and associated to the following boundary conditions:

$$
\begin{aligned}
\frac{\partial C_{p}}{\partial \zeta}(0, t ; z) & =\alpha \operatorname{Sh}(\mathrm{Gr}, z)\left[C_{p}(0, t ; z)-\left\langle C_{f}\right\rangle(z ; t)\right] \\
\frac{\partial C_{p}}{\partial \zeta}(1, t ; z) & =0
\end{aligned}
$$

and initial condition

$$
C_{p}(\zeta, 0 ; z)=1
$$

The coefficient $\alpha$ measures the strength of migration between the solid and the fluid and takes the following form

$$
\alpha=\frac{K D_{f} \Delta r}{D_{p}} .
$$

The full problem is consequently the coupling of two Cauchy problems: the first is the ordinary differential equation in space giving by Eq. (26) with initial condition (27) and the second is the partial differential equation (28) completed by the boundary conditions (29) and (30) and the initial condition (31). Three dimensionless groups are involved given by the Graetz number, Gr, the relative thickness of the solid tube, $\Delta r$, and the strength of migration $\alpha$ taking into account of the ratio of the diffusion coefficient in the liquid to the diffusion coefficient in the solid and the partition coefficient.

From the point of view of safety issues, the important quantity to know is the amount of plasticizer migrating in the liquid over a certain time. The quantity defined by equation $(25),\left\langle C_{f}\right\rangle$, determined at the exit $(z=1)$, represents the normalized mass flux of plasticizer. To know the amount of additive in the liquid, the integration over the time gives the amount of additive migration in the liquid which has flowed through the tube. So, the quantity

$$
M_{f}(t)=\int_{0}^{t}\left\langle C_{f}\right\rangle\left(1 ; t^{\prime}\right) d t^{\prime},
$$

representing the normalized amount of additive in the liquid over the time $t$ is introduced. The amount of additive over a time interval $\left[t_{1} ; t_{2}\right]$ is given by

$$
M_{f}\left(t_{1}, t_{2}\right)=\int_{t_{1}}^{t_{2}}\left\langle C_{f}\right\rangle\left(1 ; t^{\prime}\right) d t^{\prime} .
$$

The problem is solved numerically thanks to a Chebyshev-spectral method [32] to discretize equation (28) over the $\zeta$ coordinate. A backward differentiation formula at the second order [33] is used for the temporal derivative of the left hand side of (28). The axial coordinate is discretized by a uniform grid over which equation (26) is solved using a backward difference formula at the second order. The integrals (33) and (34) are determined by using a trapezoidal quadrature. For more information, details of the numerical method are given in supplementary material of this article. We provide also a study about the consistency of the numerical method by comparing the numerical results with exact solutions and using an extrapolation method to obtain the order of truncation in space and time. 


\section{Results and discussion}

Before presenting results obtained with the numerical method, values of diffusion coefficients have to be provided. The migration is investigated for an additive like a glycerol monoester plasticizer for which the diffusion coefficient has been determined by Lundsgaard et al. [23]. The typical PVC tube addressed in this work is composed with a large amount of plasticizer, around 50 weight \%. According to the previous work of [23], the diffusion coefficient of a glycerol monoester plasticizer for a temperature equal to $40^{\circ} \mathrm{C}$, is equal to $1.1 \cdot 10^{-13} \mathrm{~m}^{2} / \mathrm{s}$. According to the specifications of EU regulation, a food simulant is a liquid. For milk application, the simulant is composed of ethanol and water in the same proportion in volume. The diffusion coefficient, $D_{f}$, of the glycerol monoester plasticizer can be evaluated thanks to the Wilke and Chang's correlation [24]. For a temperature equal to $40^{\circ} \mathrm{C}$ and knowing that the molar volume of the plasticizer is equal to $500 \mathrm{~cm}^{3} / \mathrm{mol}[23], D_{f}$ is equal to $2.44 \cdot 10^{-10} \mathrm{~m}^{2} / \mathrm{s}$ meaning that the ratio $D_{f} / D_{p}$ is around $10^{3}$. Consequently, the liquid can be seen as a perfect diffuser in comparison with the solid. In the following, only one value of $D_{f} / D_{p}$ has been used taken at $10^{3}$. Various sizes of tube are investigated by changing $\Delta r$ over three decades and the Graetz number over four decades.

In the procedure dictated in [17] in support of EU directive 2002/72/EC [34], two situations have to be addressed: the former when the solubility of the plasticizer is small by taking the partition coefficient equal to $10^{-3}$ and the latter when the solubility is high corresponding to the "worst case" for which the partition coefficient is equal to 1 . In the following of this work, we will take these two limit values for $K$.

\subsection{Plasticizer concentration as a function of space and time in solid domain}

In this subsection, the behavior of $C_{p}$ in space and time is investigated. Figure $2 \mathrm{a}$ and $2 \mathrm{~b}$ depict profiles of $C_{p}$ as a function of $\zeta$ in the middle of the tube for four increasing times when the Graetz number is equal to $10^{2}$ and for partition coefficients equal to $10^{-3}$ and 1 respectively. Starting from a uniform concentration over the whole plastic domain, $C_{p}$ decreases from the solid/liquid interface when the time increases. The value of $C_{p}$ at the solid/liquid interface, i.e. for $\zeta=0$, changes strongly with $\Delta r$, underlining the coupling with the migration in the liquid. As shown in Figure 2a, the range of $C_{p}$ is very small when $\Delta r=10^{-3}$, while this range is larger and larger when $\Delta r$ increases. The partition coefficient plays also an important role in the migration. Figure $2 \mathrm{~b}$ emphasizes that the gradient of $C_{p}$ is more important when the partition coefficient is unity. When $\Delta r=10^{-3}$, the concentration profiles for increasing time present a slope more pronounced a large partition coefficient. Since the value of $C_{p}$ at the solid/liquid interface is equal to the dimensionless plasticizer concentration in the liquid, the average concentration of the plasticizer in the liquid is more important at low partition coefficient which is not obvious in the first glance and will be explained latter.

In order to study the behavior of the plasticizer concentration as a function of the axial coordinate, $C_{p}$ is given in Figure $3 \mathrm{a}$ and $3 \mathrm{~b}$ at $t=5 \cdot 10^{-3}$ in five locations over the tube length for four $\Delta r$, when the Graetz number is moderate and equal to 10 . For small $K$ and $\Delta r$, apart from the entrance, $C_{p}$ does not change strongly over the length of the tube. As already mentioned previously, the plasticizer concentration decreases at the solid/liquid interface when $\Delta r$ increases as it is shown in Figure 3a when $\Delta r$ is equal to $10^{-1}$ or 1 . The value at the solid/liquid interface increases with $z$ due to the enrichment 
(a) $K=10^{-3}$
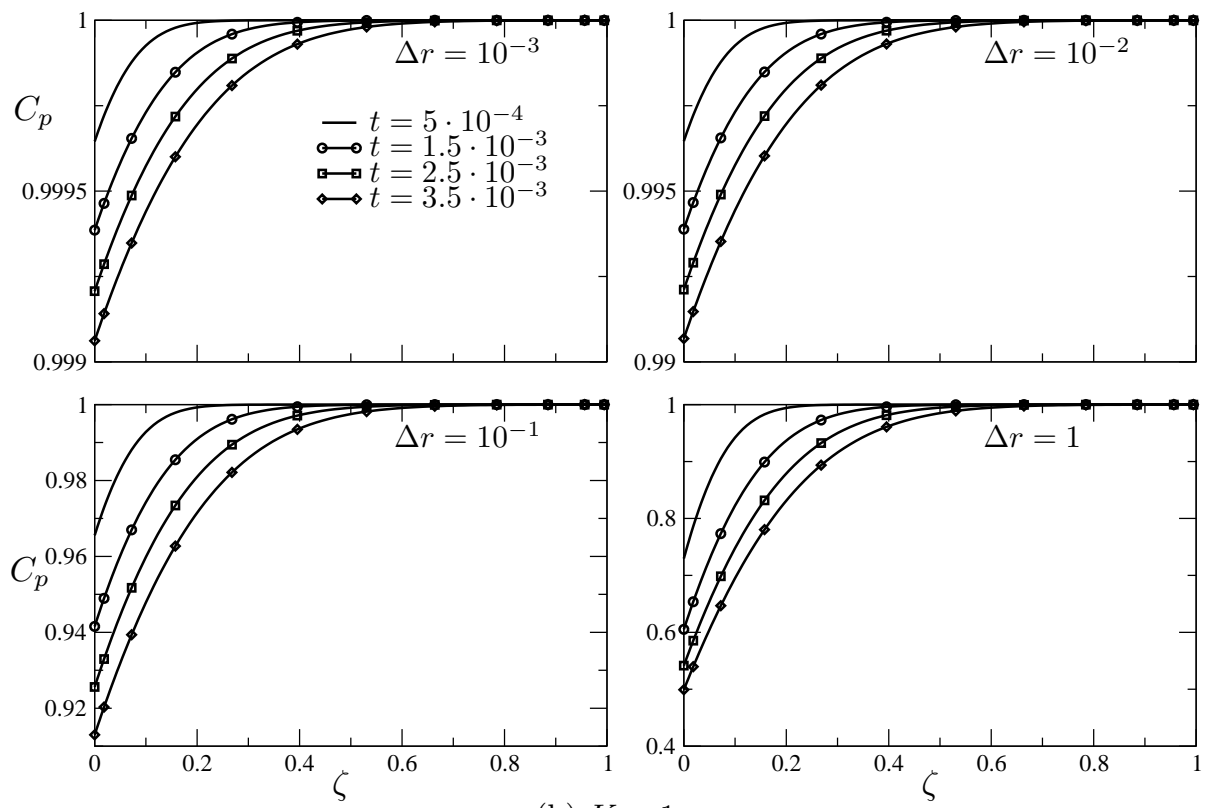

(b) $K=1$

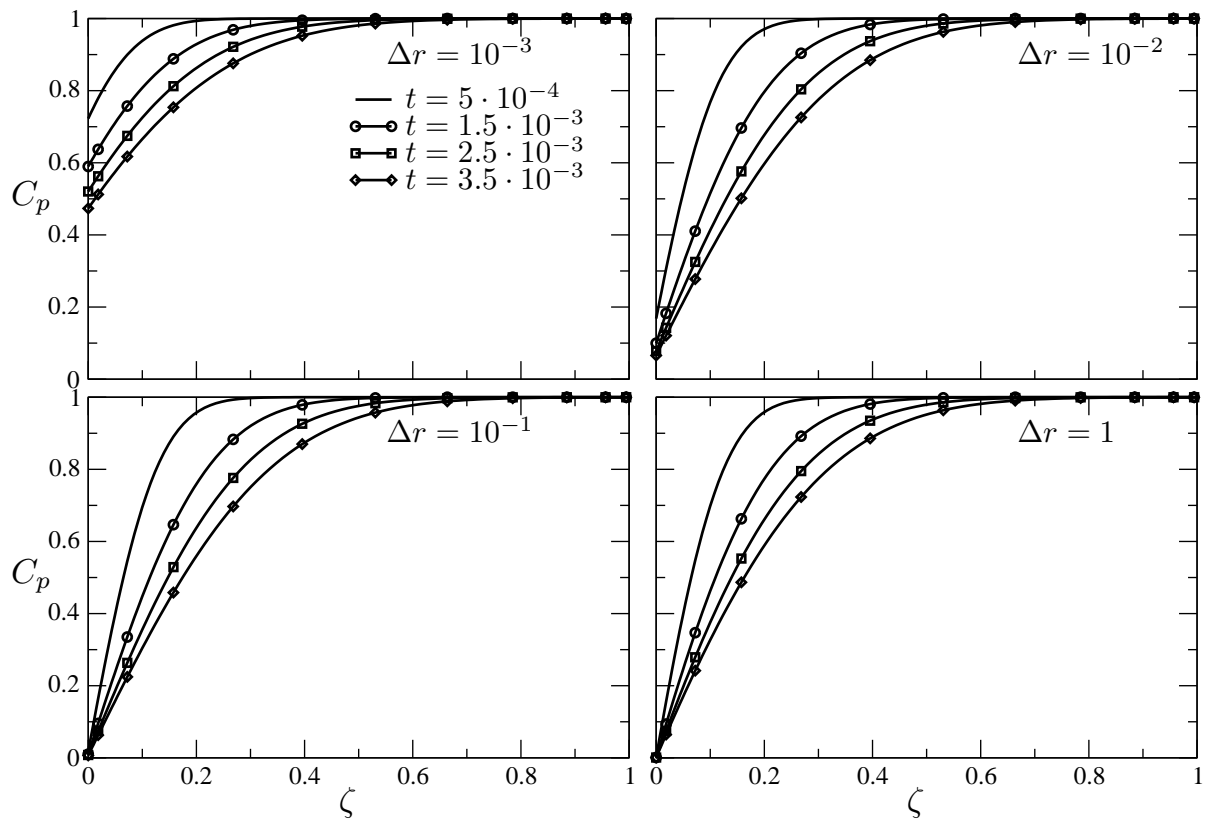

Figure 2: $C_{p}(\zeta, t ; z)$ as a function of $\zeta$ in the middle of the tube, $z=1 / 2$, when $t=5 \cdot 10^{-4}, 1.5 \cdot 10^{-3}$, $2.5 \cdot 10^{-3}$ and $3.5 \cdot 10^{-3}$ and $\mathrm{Gr}=10^{2}$ and for (a) $K=10^{-3}$ and (b) $K=1$. 
in plasticizer in the liquid phase which is clearly seen when $\Delta r=1$. The migration enhances when the partition coefficient increases (see Figure $3 \mathrm{~b}$ ). With increasing $\Delta r$, the $C_{p}$ profiles are closer and closer such as for $\Delta r=1$ the plasticizer concentrations is quasi-homogeneous over the tube length with a gradient more important.

The behavior of the plasticizer concentration in the solid shows that the influence of the tube geometry and the partition coefficient are very important in the migration process. The strength of migration is more important when the tube thickness is large due to the increase of the time scale of diffusion in the solid.

In order to study the role played by the flowing liquid, Figure $4 \mathrm{a}$ and $4 \mathrm{~b}$ present the $C_{p}$ profiles at the same time as the previous figures, i.e. $t=5 \cdot 10^{-3}$, and for the same locations but with a Graetz number equal to $10^{5}$. The partition coefficients are $K=10^{-3}$ in Figure $4 \mathrm{a}$ and $K=1$ in Figure $4 \mathrm{~b}$. With the increase of Gr, the plasticizer concentration at the solid/liquid interface decreases significantly. Inversely to the previous case where the Graetz number was equal to 10 , the influence of $\Delta r$ is least. Here, we pointed out the strong influence of the advection process in the migration dynamics. Indeed, as it is can be seen on the equation describing the behaviour of $\left\langle C_{f}\right\rangle$ as a function of $z$, eq. (26), we expect a decrease of $\left\langle C_{f}\right\rangle$ when the Graetz number increases even if the Sherwood number rises up with Gr. These results point out that when the flow rate increases the concentration in the liquid decreases limiting the migration process.

The partition coefficient plays a significant role as it is shown in Figure 4b. Even for a small $\Delta r$, the plasticizer concentration is quasi-homogeneous over the length of the tube, which is not the case when the partition coefficient is small, see Figure 4a when $\Delta r=10^{-3}$. The comparison of Figure $4 \mathrm{a}$ and $4 \mathrm{~b}$ emphasizes also that the migration dynamics is driven by the partition coefficient. When the partition coefficient is small, the plasticizer concentration in the solid domain stays uniformly equal to one in a large part of the thickness. Conversely, for a partition coefficient equal to one, $C_{p}$ varies over the whole range of $\zeta$ at the same time.

Note that the role played by $K$ and $\Delta r$ are quite similar. Numerical results show that the plasticizer concentration in the tube is more and more homogeneous over the length of the tube for both large values of $K$ and $\Delta r$. This similarity in the behavior played by $K$ and $\Delta r$ comes from the fact that these two quantities are both factors of the coefficient $\alpha$ given by equation (32). When $\alpha$ is large, the boundary condition (29) shows that $C_{p}(\zeta=0, t ; z)$ should be close to the average concentration of plasticizer in the liquid. Conversely, in the limit of small $\alpha$, the flux of $C_{p}$ at the solid/liquid interface decreases as it is clearly shown in Figures 3a when $\Delta r$ is equal to $10^{-3}$ or $10^{-2}$. In conclusion, the larger $\alpha$, the larger the migration, meaning that the concentration of plasticizer in the liquid increases when both $K$ and $\Delta r$ increase. This result seems realizable for large $\Delta r$ since the dilution decreases with the radius ratio. The role of the partition coefficient is less obvious. It is understandable that when $K$ is large, the gradient of plasticizer concentration should be more pronounced.

It is noteworthy that $C_{p}$ versus $\zeta$ behaves equivalently in the entrance of the hose whatever values of $K$ and $\Delta r$. The plasticizer concentration at the solid/liquid interface is equal to zero due to the fact that the Sherwood number has a singular behavior at the entrance where Sh diverges to infinity. Since the fluid enters the tube with an average concentration of plasticizer equal to zero, the boundary condition (29) implies that the concentration at the solid/liquid interface is equal to zero. This fact has been used to control the numerical accuracy since in this particular case, an exact solution can be 
(a) $K=10^{-3}$
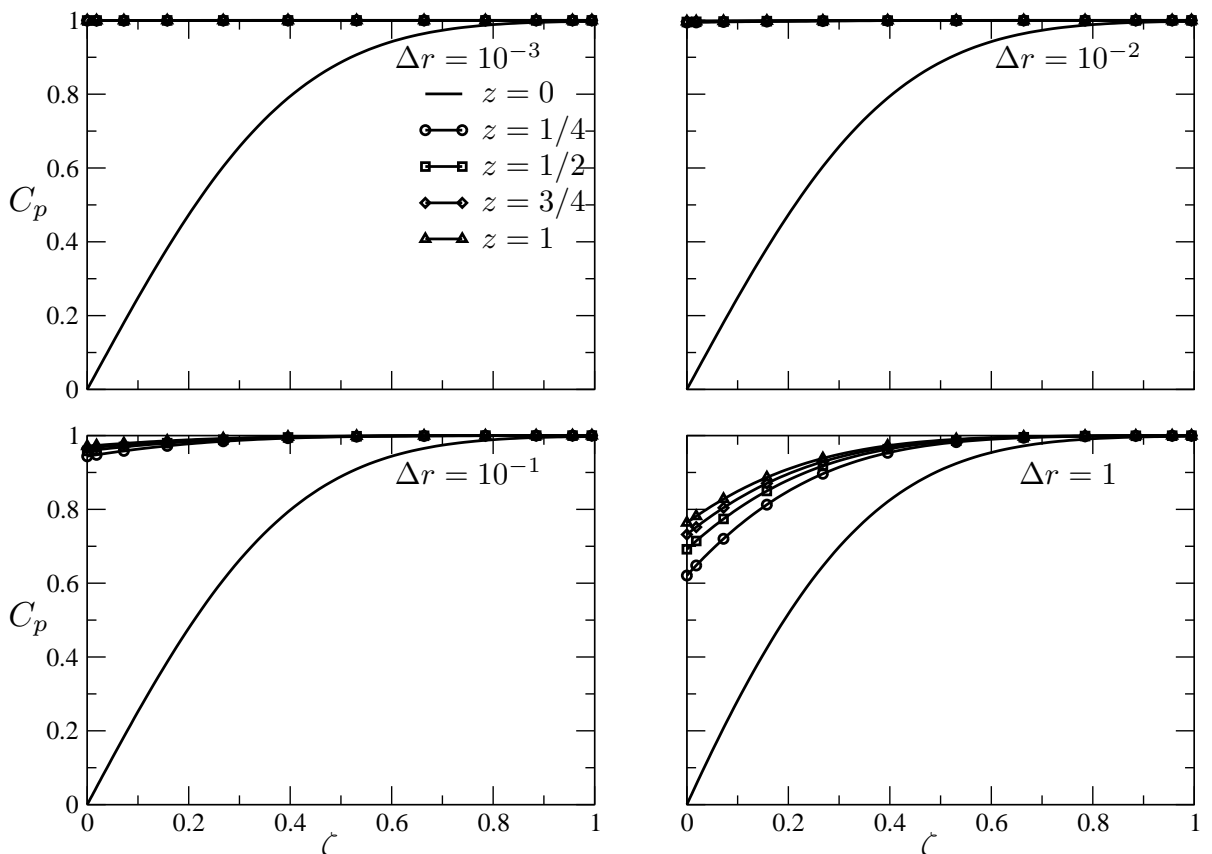

(b) $K=1$
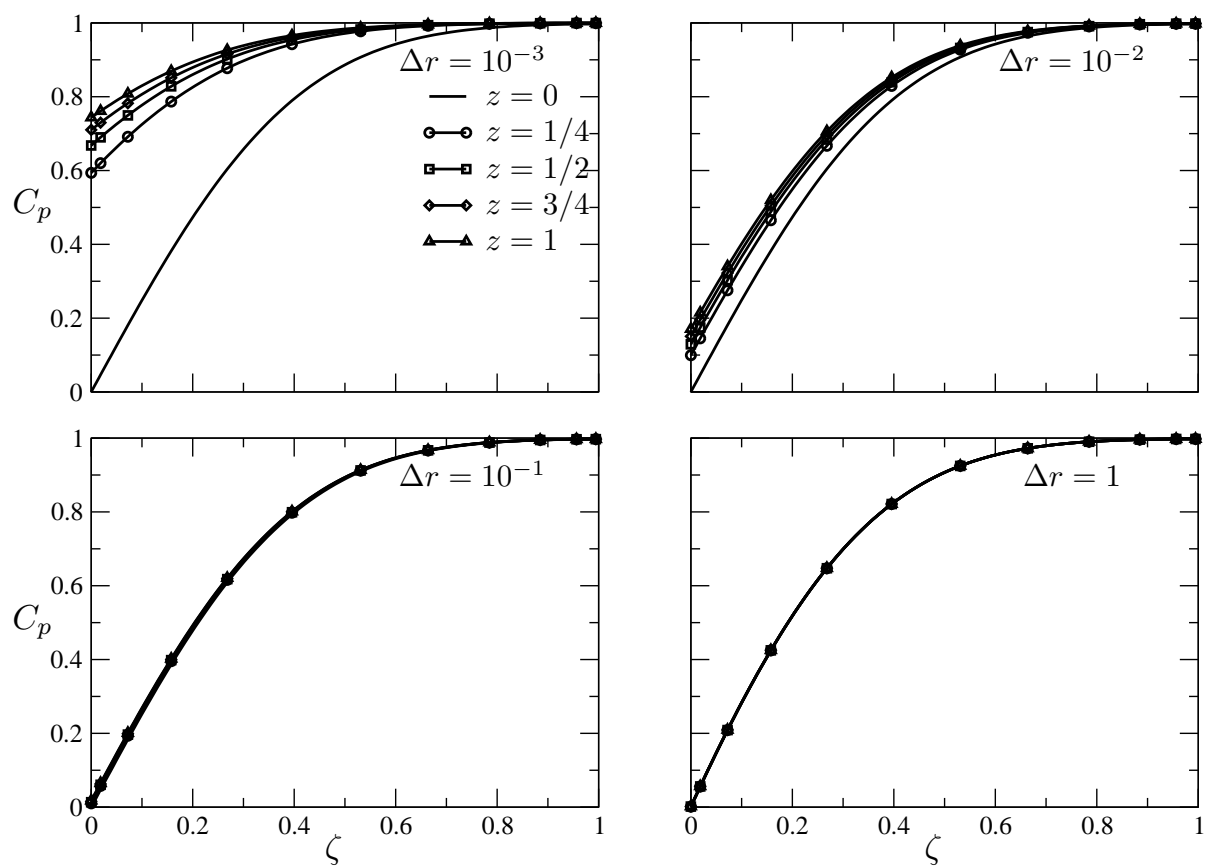

Figure 3: $C_{p}(\zeta, t ; z)$ as a function of $\zeta$ when $t=5 \cdot 10^{-3}$ and for five positions in the tube such as $z=0$, $1 / 4,1 / 2,3 / 4$ and 1 when the Graetz number is equal to 10 and for (a) $K=10^{-3}$ and (b) $K=1$. 
(a) $K=10^{-3}$
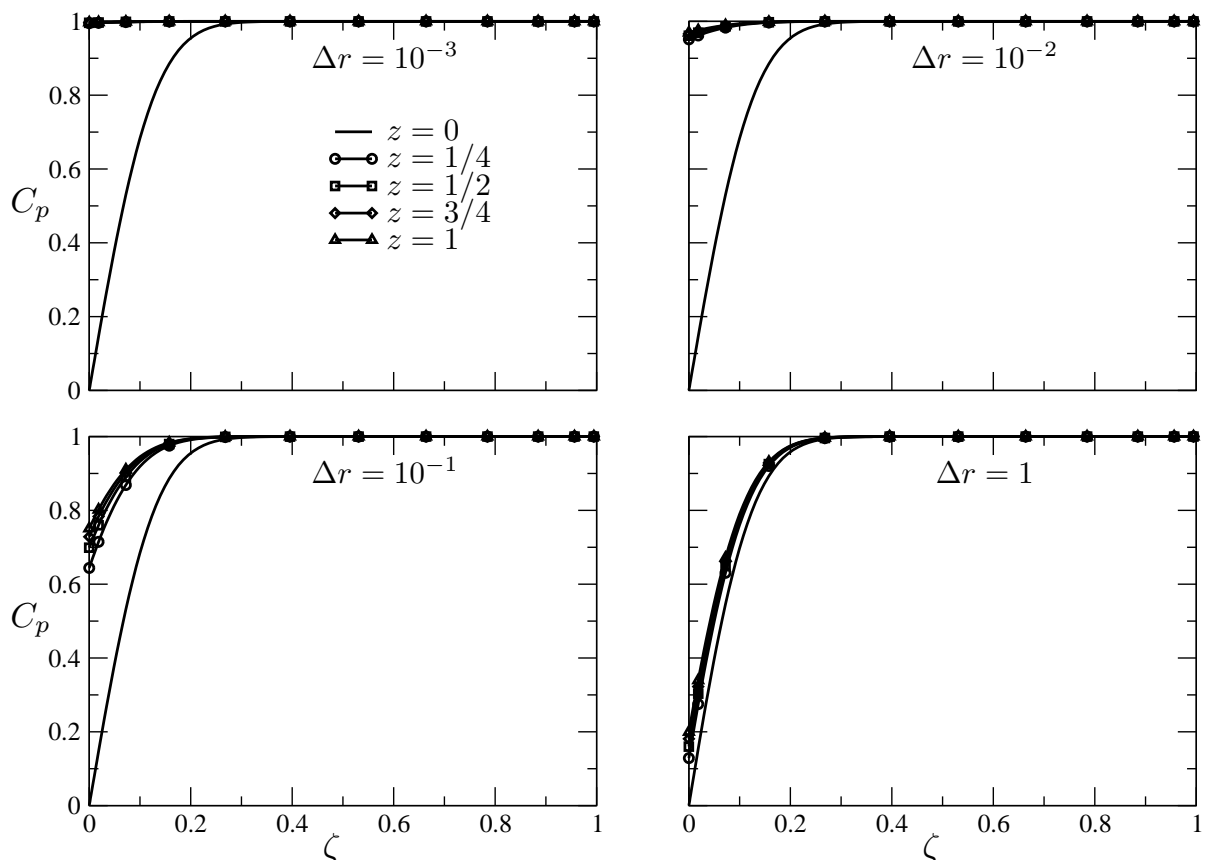

(b) $K=1$
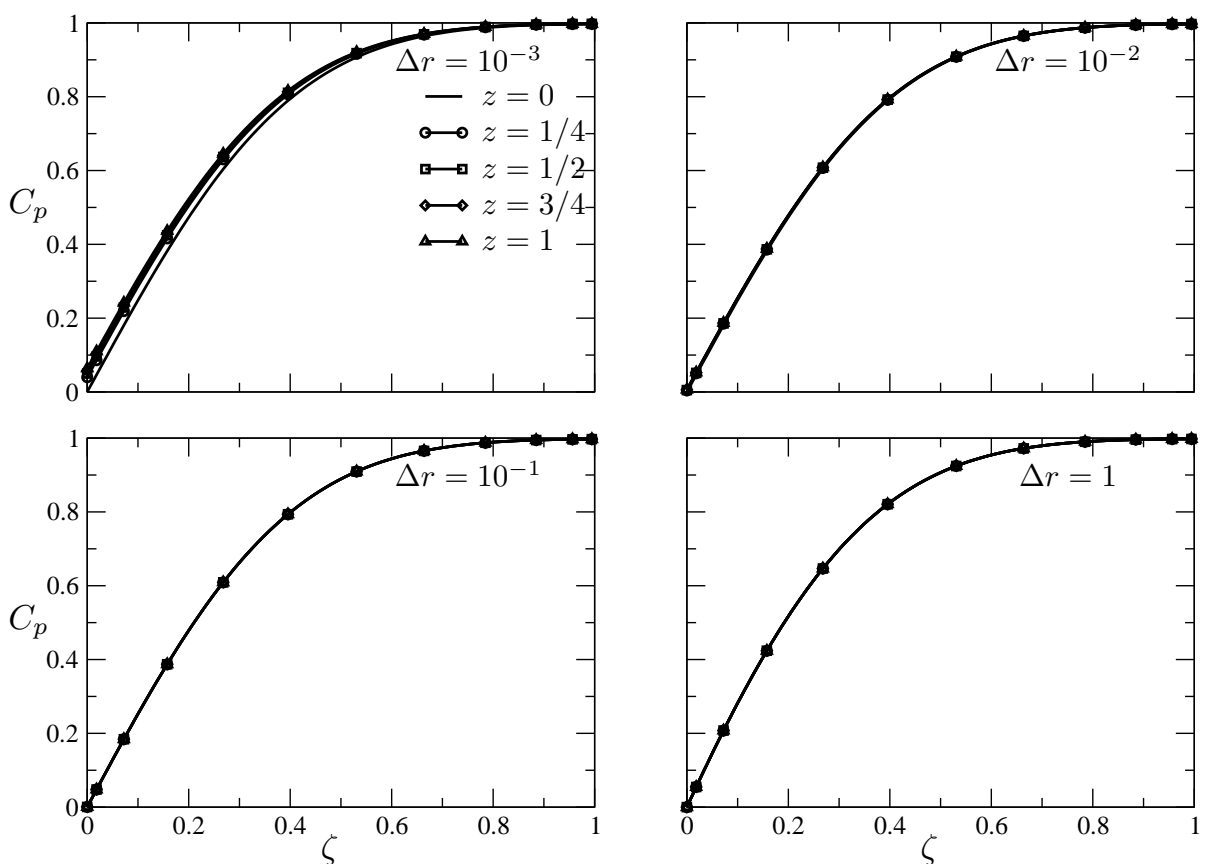

Figure 4: $C_{p}(\zeta, t ; z)$ as a function of $\zeta$ when $t=5 \cdot 10^{-3}$ and for five positions in the tube such as $z=0$, $1 / 4,1 / 2,3 / 4$ and 1 when the Graetz number is equal to $10^{5}$ and for (a) $K=10^{-3}$ and (b) $K=1$. 
easily established (see supplementary material).

\subsection{Plasticizer concentration as a function of $z$ and time in liquid domain}

Now let us turn to the behavior of the plasticizer concentration in the liquid. As previously done, the combined roles of the tube dimension, the partition coefficient and the Graetz number are provided. Figure 5a presents profiles of $\left\langle C_{f}\right\rangle$ as a function of $z$ for four values of $\Delta r$ and for five times increasing with a time step equal to $2.5 \cdot 10^{-3}$ and for a Graetz number equal to 10 . The partition coefficient is taken at a small value equal to $10^{-3}$. Remark that at the initial time, $\left\langle C_{f}\right\rangle$ is not a uniform profile over the tube length because, at the time zero, the liquid is instantaneous in motion throughout the tube. At first glance, this result is questionable. It is due to the fact that in Quasi-Steady State Approximation, the average of plasticizer concentration in the liquid does not depend on the time. We point out in problem statement ( $(2)$ that $t$ is only a parameter of the problem in the liquid domain. Concentration $\left\langle C_{f}\right\rangle$ depends only upon the axial coordinate. It is a solution of the Cauchy problem for which an initial condition is set at the entrance of the tube. Consequently, profiles plotting at $t=0$ correspond to the solution obtained in steady state regime established over a time proportional to the residence time of the liquid in the tube which is always very small in comparison with the diffusion time scale of the plasticizer in the solid domain. The behavior of $\left\langle C_{f}\right\rangle$ versus $z$ reflects the change of the Sherwood number when $z$ increases. Close to the entrance, Sh is important leading to a strong increase of $\left\langle C_{f}\right\rangle$ which is attenuated with the increasing location in the tube. The average concentration of plasticizer decreases with time due to the drop of the plasticizer concentration at the solid/liquid interface. The decrease of $\left\langle C_{f}\right\rangle$ with time enhances with $\Delta r$. Since the amplitude of $\left\langle C_{f}\right\rangle$ decreases with the coefficient $\alpha$, the increase of $\Delta r$ leads to the decrease of $\left\langle C_{f}\right\rangle$. Nevertheless, for this particular Graetz number, the decrease is slight.

In Figure 5b, the average concentration of plasticizer in the liquid is plotted as a function of $z$ for five times increasing with a time step equal to $2.5 \cdot 10^{-3}$ when the partition coefficient is taken equal to 1 and $\mathrm{Gr}=10$. In comparison with the previous results obtained for $K$ equal to $10^{-3}$, the influence of the variation of the Sherwood number seems less important.

As it is shown in the behavior of $C_{p}$, the thickness of the tube plays an important role on the plasticizer concentration in the liquid. Figure $5 \mathrm{~b}$ shows that $\left\langle C_{f}\right\rangle$ decreases more and more when $\Delta r$ increases. Moreover, it is noteworthy that the slope of the profile of $\left\langle C_{f}\right\rangle$ which is important for small $\Delta r$ close to the tube entrance becomes practically constant over the tube length for large values of $\Delta r$ giving linear profiles of $\left\langle C_{f}\right\rangle$ as a function of $z$. The comparison of results at $\Delta r=10^{-1}$ and $\Delta r=1$ underlines that the average plasticizer concentration in the liquid loses one order of magnitude while $\Delta r$ is multiplied by 10 .

To see the influence of the advection, Figures $6 \mathrm{a}$ and $6 \mathrm{~b}$ present the average plasticizer concentration in the liquid as a function of $z$ when the Graetz number is equal to $10^{5}$ and for $K=10^{-3}$ and 1 respectively. The effect of liquid motion is clearly established since the additive concentration in the liquid decreases significantly when the Graetz number increases for four orders of magnitude. In Figure $6 \mathrm{~b}$ for which $K=1,\left\langle C_{f}\right\rangle$ behaves linearly with $z$. As already pointed out above, the increase of one order of magnitude of $\Delta r$ leads to a decrease of one order of magnitude of $\left\langle C_{f}\right\rangle$ showing that $\left\langle C_{f}\right\rangle$ is inversely proportional to the $\alpha$ coefficient. 
(a) $K=10^{-3}$

$\left\langle C_{f}\right\rangle$
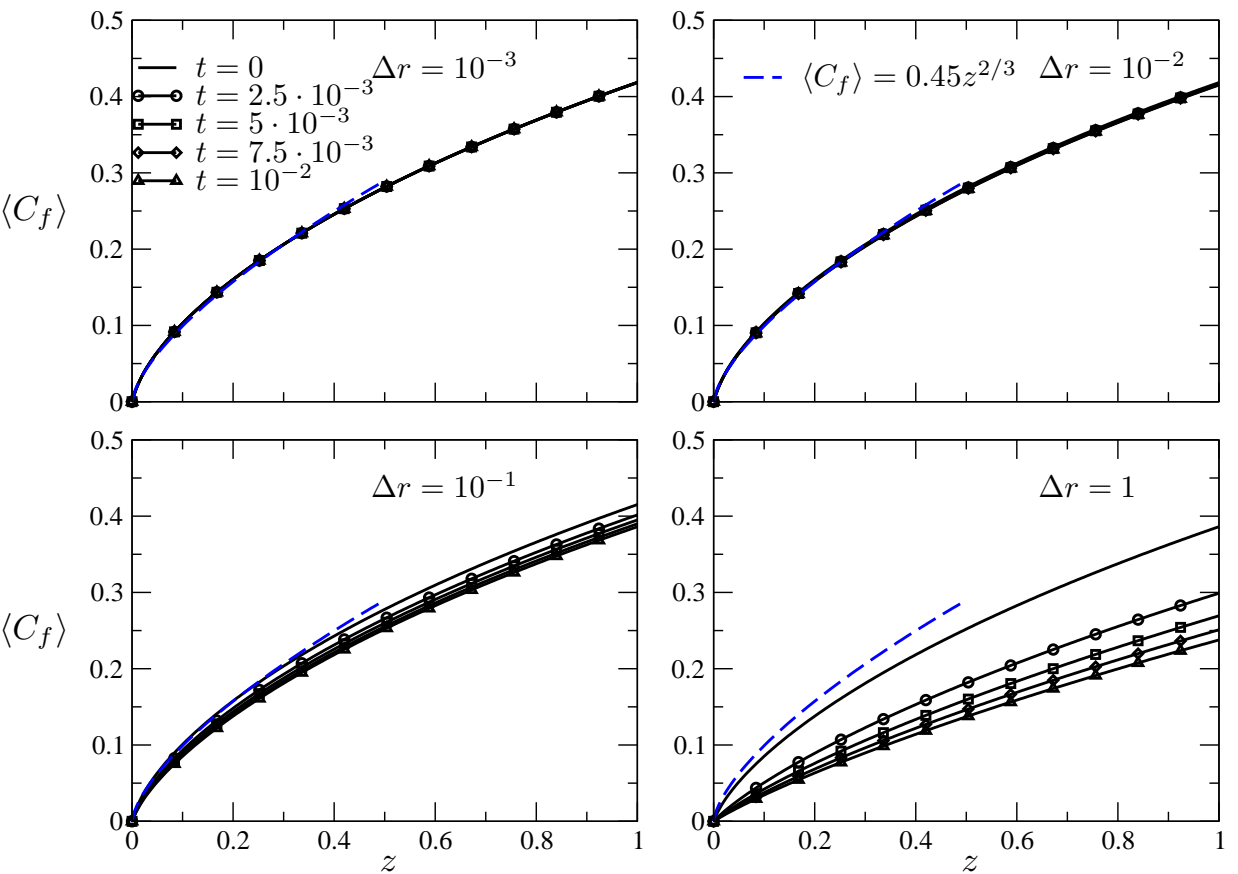

(b) $K=1$
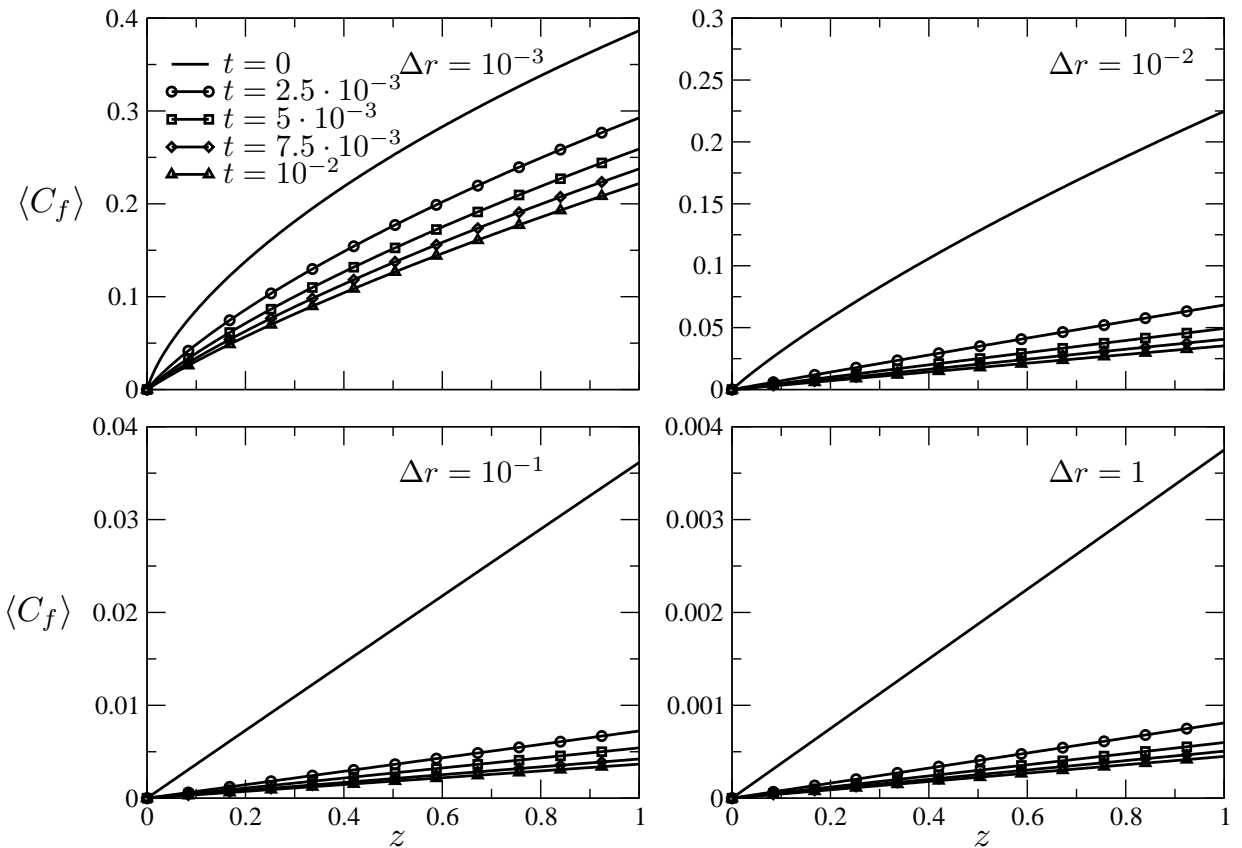

Figure 5: $\left\langle C_{f}(z ; t)\right\rangle$ as a function of $z$ when the Graetz number is equal to 10 at the times equal to $t=0$, $2.5 \cdot 10^{-3}, 5 \cdot 10^{-3}, 7.5 \cdot 10^{-3}$ and $10^{-2}$ and for (a) $K=10^{-3}$ and (b) $K=1$. 
(a) $K=10^{-3}$
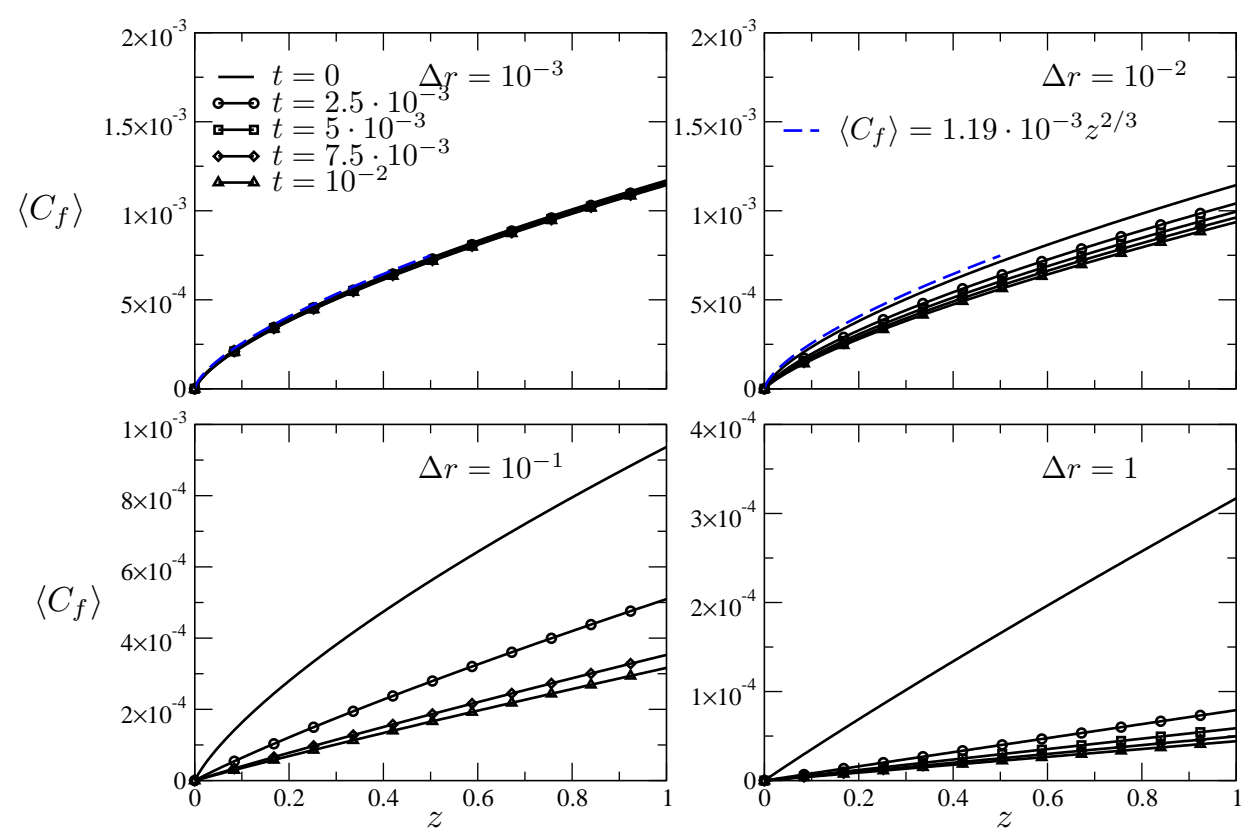

(b) $K=1$
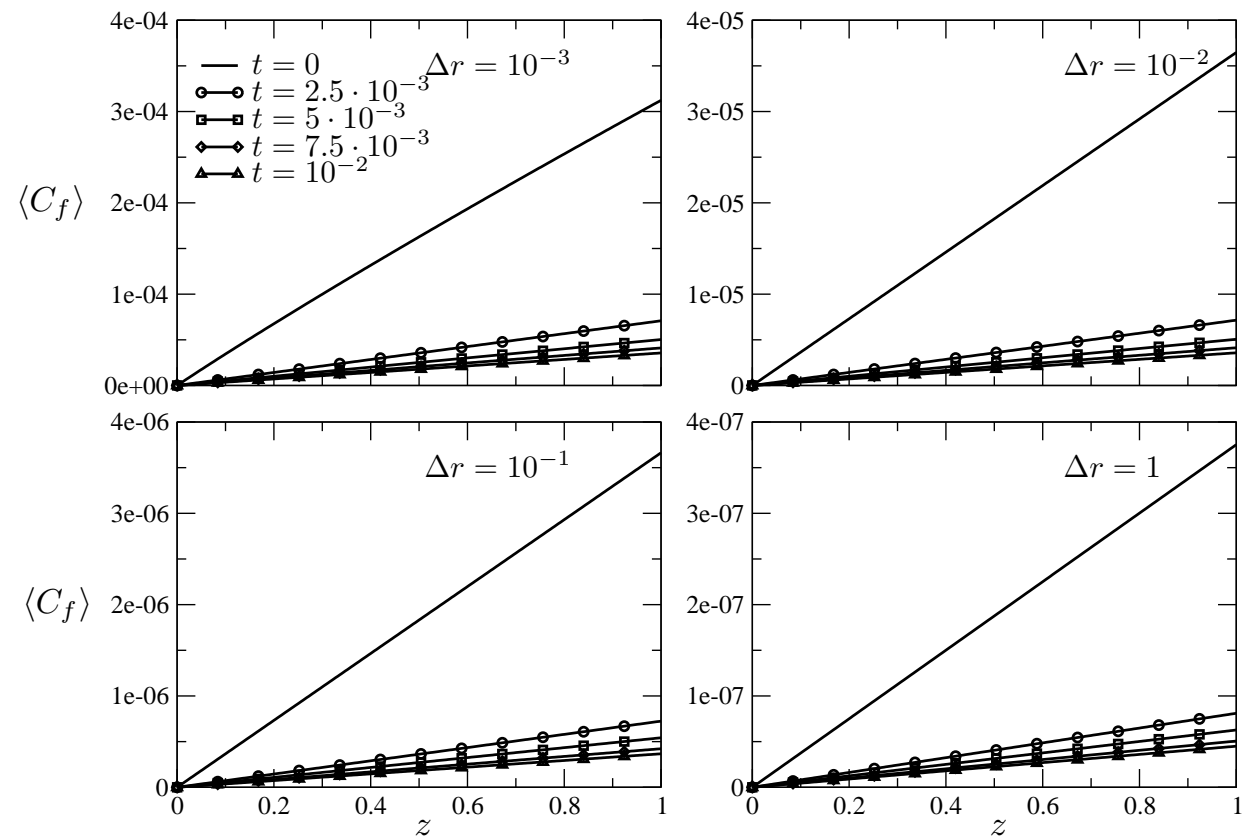

Figure 6: $\left\langle C_{f}(z ; t)\right\rangle$ as a function of $z$ when the Graetz number is equal to $10^{5}$ at the times $t=0$, $2.5 \cdot 10^{-3}, 5 \cdot 10^{-3}, 7.5 \cdot 10^{-3}$ and $10^{-2}$ and for (a) $K=10^{-3}$ and (b) $K=1$. 
The decrease of the partition coefficient plays an important role in the behavior of $\left\langle C_{f}\right\rangle$ even if the Graetz number is important as it is seen in Figure 6a. At small $\Delta r$, the average plasticizer concentration in the liquid is larger than for $K=1$. Moreover, the curvature of the profile is more important. We point out that $\left\langle C_{f}\right\rangle$ does not change significantly over the time chosen to represent the various profiles. As already pointed out, when the radius ratio $\Delta r$ increases, the $\left\langle C_{f}\right\rangle$ profiles are quasi-linear as a function of $z$. The proportionality with the inverse of $\alpha$ is very well verified when $\Delta r=1$ since the comparison of Figures $6 \mathrm{~b}$ and $6 \mathrm{a}$ shows that the $\left\langle C_{f}\right\rangle$ profiles are very similar as a function of $z$ and decrease equivalently when the time increases apart from the amplitude of $\left\langle C_{f}\right\rangle$ obtained for $K=10^{-3}$ is three orders of magnitude larger than for $K=1$.

The linear behavior of $\left\langle C_{f}\right\rangle$ as a function of $z$ when $\alpha$ is larger than one can be easily demonstrated. Indeed, from the boundary condition on $C_{p}$ written at the solid/liquid interface, i.e. $\zeta=0$ (eq. 29), the difference $C_{p}(0, t ; z)-\left\langle C_{f}\right\rangle(z ; t)$ behaves like

$$
C_{p}(0, t ; z)-\left\langle C_{f}\right\rangle(z ; t)=\frac{1}{\alpha \operatorname{Sh}(\mathrm{Gr}, z)} \frac{\partial C_{p}}{\partial \zeta}(0, t)
$$

Reporting this relation in the ordinary differential equation describing the behavior of $\left\langle C_{f}\right\rangle(z ; t)$, Eq. (26), gives us the following relation

$$
\frac{d\left\langle C_{f}\right\rangle}{d z}=\frac{2}{\operatorname{Gr} \alpha} \frac{\partial C_{p}}{\partial \zeta}(0, t)
$$

showing that the slope of $\left\langle C_{f}\right\rangle(z ; t)$ is independent of $z$ since the Sherwood number does not appear in the previous relation. It is noteworthy that the slope of $\left\langle C_{f}\right\rangle$ obtained from the previous relation is inversely proportional to $\alpha$.

In the opposite situation when $\alpha$ is much smaller than one, the difference $C_{p}(0, t ; z)-$ $\left\langle C_{f}\right\rangle(z ; t)$ should be finite in order to avoid the simplest solution of $\left\langle C_{f}\right\rangle(z ; t)$ independent of $z$. So, the boundary condition written in $\zeta=0$, Eq. (29), implies that the normal derivative of $C_{p}$ should be proportional to $\alpha \mathrm{Sh}(\mathrm{Gr}, z)$. Consequently, the difference $C_{p}(0, t ; z)-\left\langle C_{f}\right\rangle(z ; t)$ is constant and independent of $z$. Since when the Graetz number is larger than one, the Sherwood number is inversely proportional to $z^{1 / 3}$. The integration of Eq. (26) with $z$ shows that $\left\langle C_{f}\right\rangle(z ; t)$ is proportional to $z^{2 / 3}$. This behavior is very well verified in Figure 6a obtained numerically for $K=10^{-3}$ and $\mathrm{Gr}=10^{5}$ for $\Delta r=10^{-3}$ for which an approximate solution proportional to $z^{2 / 3}$ has been plotted in blue solid line.

The behavior of $\left\langle C_{f}\right\rangle$ underlines two important results. Firstly, the average concentration of plasticizer in the liquid decreases with time meaning that there is not accumulation in the liquid phase. This decrease of $\left\langle C_{f}\right\rangle$ stems from the decrease of $C_{p}$ at the solid/liquid interface. Secondly, when the Graetz number increases, $\left\langle C_{f}\right\rangle$ decreases due to the reduction of residence time of the liquid over the tube length even if the Sherwood number increases with the Graetz number.

\subsection{Behavior of $M_{f}$ versus of time and comparison with static condition}

The amount of plasticizer migrating in the liquid over a certain time is given by the quantity $M_{f}$ defined by equation (33), and is important to know for safety issues. In order to compare with the static condition, the conjugated migration problem has been stated and solved numerically thanks to a coupled spectral method both used for the 
liquid and solid domains. Details about the problem statement in static condition are given in Appendix A.

Figure 7a represents $M_{f}(t)$ versus time both in static and dynamic conditions when the partition coefficient is equal to $10^{-3}$ for $\Delta r$ equal to $10^{-3}, 10^{-2}, 10^{-1}$ and 1 and Graetz numbers equal to $10,10^{2}, 10^{3}, 10^{4}$ and $10^{5}$. The solid lines represent the solution obtained in static condition, which is determined by equation (A.8) in Appendix A. As expected from the previous analysis on $\left\langle C_{f}\right\rangle$, the larger the Graetz number, the smaller the amount of plasticizer in the liquid. It is noteworthy that for small radius ratio, the migration into the liquid in a static condition is smaller than in dynamic condition when the Graetz number is smaller than 10 at long times. However, when $\Delta r$ is greater or equal to $10^{-2}$, the amount of plasticizer in the liquid in dynamic condition is always smaller than in static condition for any Graetz number. In the static condition, the level of migration is more important so that the steady state is reached when the radius ratios are equal to $10^{-1}$ and 1 .

An algebraic behavior is observed for both static and dynamic conditions far away from the equilibrium state when the partition coefficient is equal to $10^{-3}$. In this particular situation and when the radius ratio stays small, i.e. lesser or equal to $10^{-1}, M_{f}$ behaves linearly with time. However, remark that for the largest $\Delta r$, the exponent of the power law decreases as a function of the Graetz number.

Figure 7b presents the profiles of $M_{f}(t)$ as a function of $t$ for same $\Delta r$ and Gr when $K=1$. In static condition, the level of migration is smaller than this one observed when the partition coefficient is equal to $10^{-3}$. As already seen in Figure 7a, an algebraic law arises from the numerical results depending of the radius ratio both for the static and dynamic conditions. For small value of $\Delta r, M_{f}$ grows up as a square root of time in static situation while in dynamic condition, $M_{f}$ behaves linearly with time. Remark that the behavior in square root of time is observed whatever the radius ratio in static condition. Conversely, the exponent of the algebraic law changes as a function of $\Delta r$ in dynamic condition. Indeed, when $\Delta r=10^{-1}$ or $1, M_{f}$ behaves as a square root of time whatever the Graetz number. Profiles obtained for various values of $\mathrm{Gr}$ form a parallel network of curves for which an increase of one order of magnitude of the Graetz number leads to a decrease of one order of magnitude of $M_{f}$.

\subsection{Specific migration for periodic working}

In practical applications, the liquid flows through tubes intermittently. For instance in milk production, the milking takes around 10 minutes a cow for an amount of milk around 10 litres. Consequently, the typical flow rates are around $1 \mathrm{l} / \mathrm{min}$. The tube sizes used for this kind of application are an interior and exterior radii equal to $7.9375 \cdot 10^{-3}$ and $1.27 \cdot 10^{-2} \mathrm{~m}$ respectively corresponding to $5 / 8$ and 1 inch in diameter. In the following, the migration has been determined for periodic working with a time period equal to $T=10 \mathrm{~min}$ and a flow rate of $1 \mathrm{l} / \mathrm{min}$. The amount of plasticizer migrating through the liquid is determined thanks to the relation (34). The specific migration of the plasticizer corresponds to the amount of plasticizer in the liquid after one period of contact divided by the total mass of liquid. In dynamic conditions, the specific migration, given in $\mathrm{mg} / \mathrm{kg}$, for a period $p$ can be determined from the dimensionless quantity $M_{f}\left(t_{1}, t_{2}\right)$ as follows

$$
\operatorname{SM}_{\mathrm{dyn}}(p)=\frac{K C_{p, 0}}{T \rho_{\mathrm{liq}}} \frac{\Delta r^{2}}{D_{p}} M_{f}[(p-1) T, p T] \cdot 10^{6},
$$


(a) $K=10^{-3}$
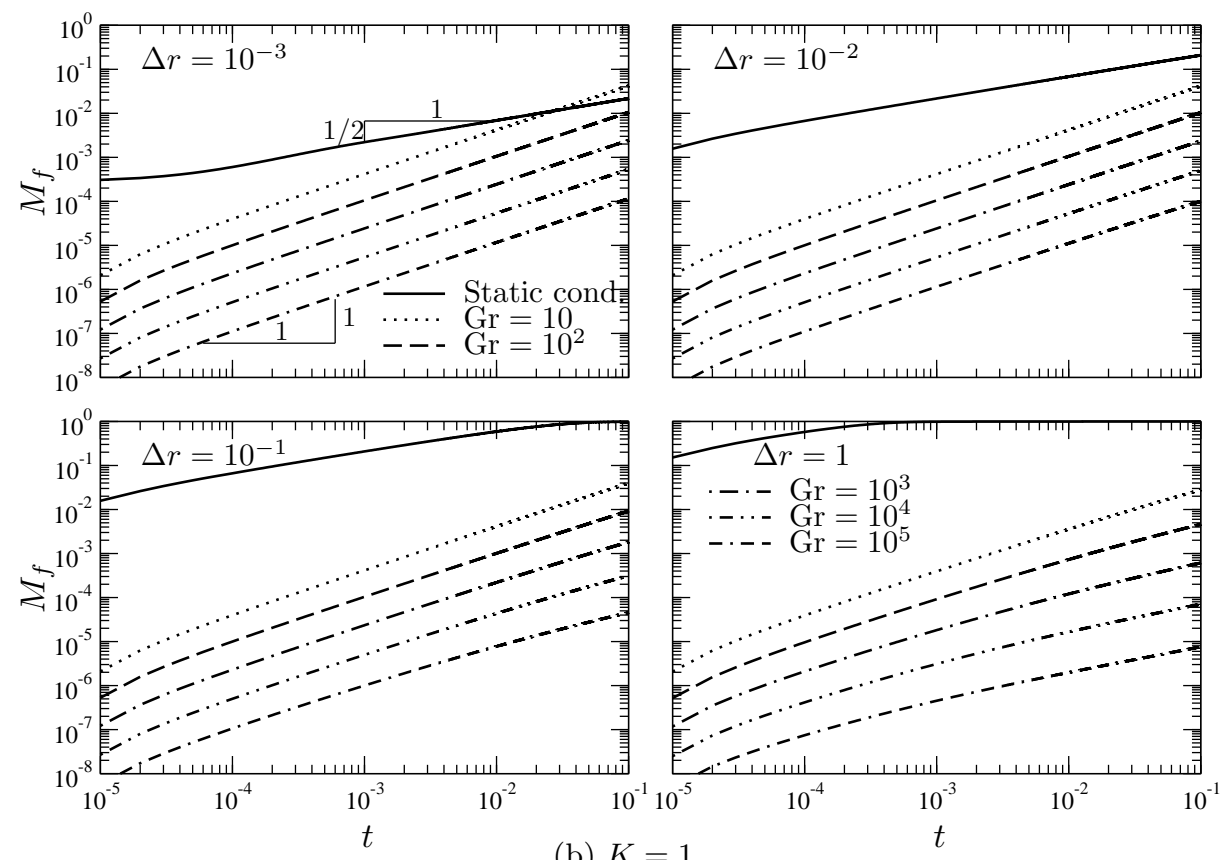

(b) $K=1$
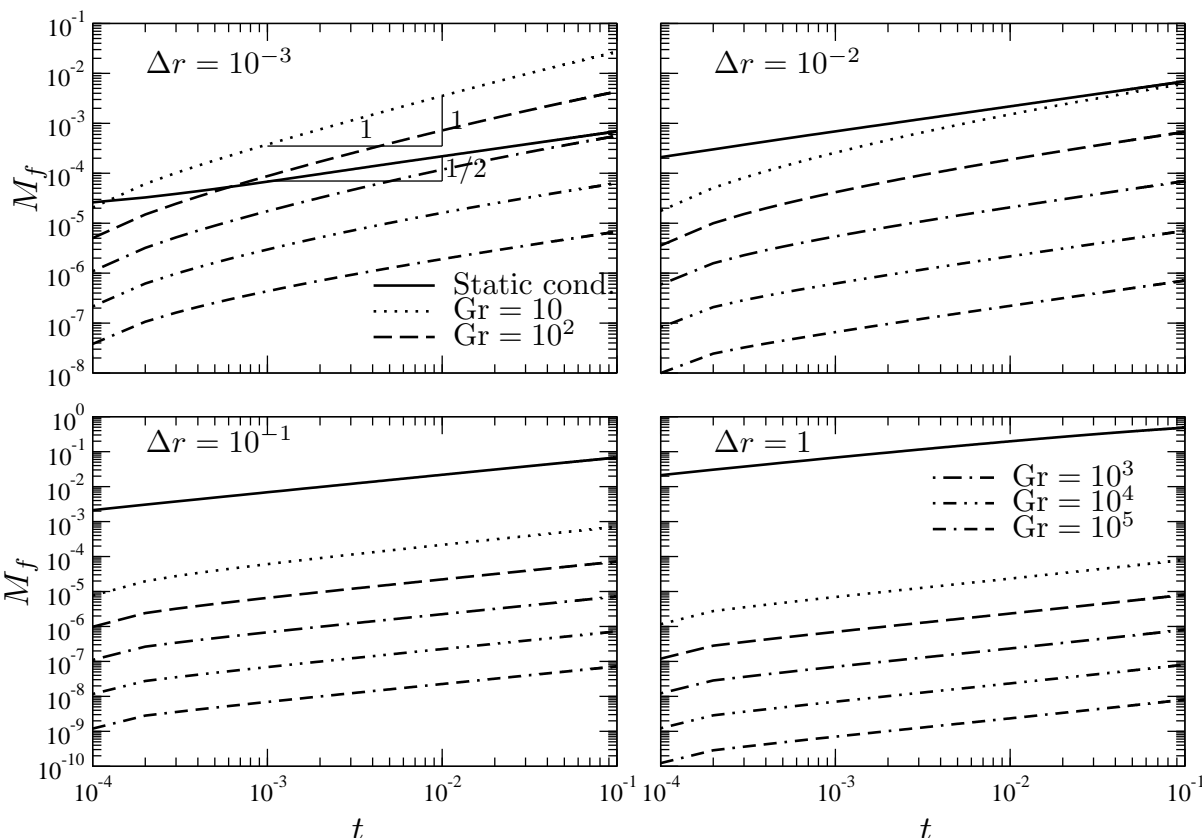

Figure 7: $M_{f}(t)$ as a function of time for four $\Delta r$ and five Graetz numbers for (a) $K=10^{-3}$ and (b) $K=1$. The solid lines depict the solution in static condition. 
(a) Liquid properties

\begin{tabular}{|c|c|c|}
\hline$\rho_{\text {liq }}\left(\mathrm{kg} / \mathrm{m}^{3}\right)$ & $D_{f}\left(\mathrm{~m}^{2} / \mathrm{s}\right)$ & $\mu(\mathrm{Pa} \cdot \mathrm{s})$ \\
\hline 914 & $2.44 \cdot 10^{-10}$ & $1.59 \cdot 10^{-3}$ \\
\hline
\end{tabular}

(b) Plasticizer properties

\begin{tabular}{|c|c|}
\hline$C_{p, 0}\left(\mathrm{~kg} / \mathrm{m}^{3}\right)$ & $D_{p}\left(\mathrm{~m}^{2} / \mathrm{s}\right)$ \\
\hline 605 & $1.1 \cdot 10^{-13}$ \\
\hline
\end{tabular}

Table 1: Physical properties of the plasticizer and the liquid used in the numerical computations of periodic working in static and dynamic conditions.

\begin{tabular}{|c|c|c|c|}
\hline L (m) & 2 & 4 & 8 \\
\hline Gr, Eq. (19) & 10871 & 5435.6 & 2717.8 \\
\hline
\end{tabular}

Table 2: Graetz numbers determined by equation (19) for the three hose lengths taken to compute the specific migrations.

in which $\rho_{\text {liq }}$ is the liquid density. Note that in the last equation $\Delta r$ is written in physical dimension. The flow rate does not appear in the last relation because the plasticizer migration is a quantity normalized by the flow rate and the amount of liquid is proportional to $Q$.

The dynamic working is once again compared to the static working for which the specific migration is given by

$$
\operatorname{SM}_{\text {stat }}(p)=\frac{K C_{p, 0}}{\rho_{\text {liq }}}\left\{\left\langle C_{f}\right\rangle(p T)-\left\langle C_{f}\right\rangle[(p-1) T]\right\} \cdot 10^{6} .
$$

The numerical applications have been done for a PVC tube with $50 \%$ of a glycerol monoester plasticizer and a liquid corresponding to a mixing of $50 \%$ of ethanol in water. The physical properties taken for the numerical computations are summarized in Table 1. The diffusion coefficient of the plasticizer in the liquid has been determined as previously from the Wilke and Chang's correlation [24]. The properties for the water/ethanol mixture have been taken from the reference [35]. With the flow rate of $1 \mathrm{l} / \mathrm{min}$ and the interior diameter of $1.5875 \cdot 10^{-2} \mathrm{~m}$, the Reynolds number is equal to 767.17 meaning that the flow regime is laminar.

The numerical computations have been achieved for three hose lengths equal to 2 , 4 , and $8 \mathrm{~m}$ which gives Graetz numbers given in Table 2. As previously, two partition coefficients have been taken: the former is a small value equal to $10^{-3}$ and the latter is $K=1$ which can be considered as the "worst case". The specific migration has been computed for ten periods. Figure 8a depicts the specific migrations in static and dynamic conditions for $K=10^{-3}$ and Figure $8 \mathrm{~b}$ for $K=1$. The specific migration limit (SML) for the plasticizer is set equal to $60 \mathrm{mg} / \mathrm{kg}$. This threshold has been plotted in solid line in Figures 8a and 8b.

When the partition coefficient is small, the level of the specific migration is close to the SML. Apart from the first value obtained in static condition, specific migration both in static and dynamic conditions is below the SML. Nevertheless, the specific migration in dynamic condition is much smaller than this obtained in static condition. For the largest Graetz number, the order of magnitude of the specific migration is around 3 $\mathrm{mg} / \mathrm{kg}$ which is smaller than one order of magnitude of the SML. Moreover, remark that the specific migration decreases with the period slowly in dynamic condition while the decrease is more pronounced in static condition. 
(a) $K=10^{-3}$

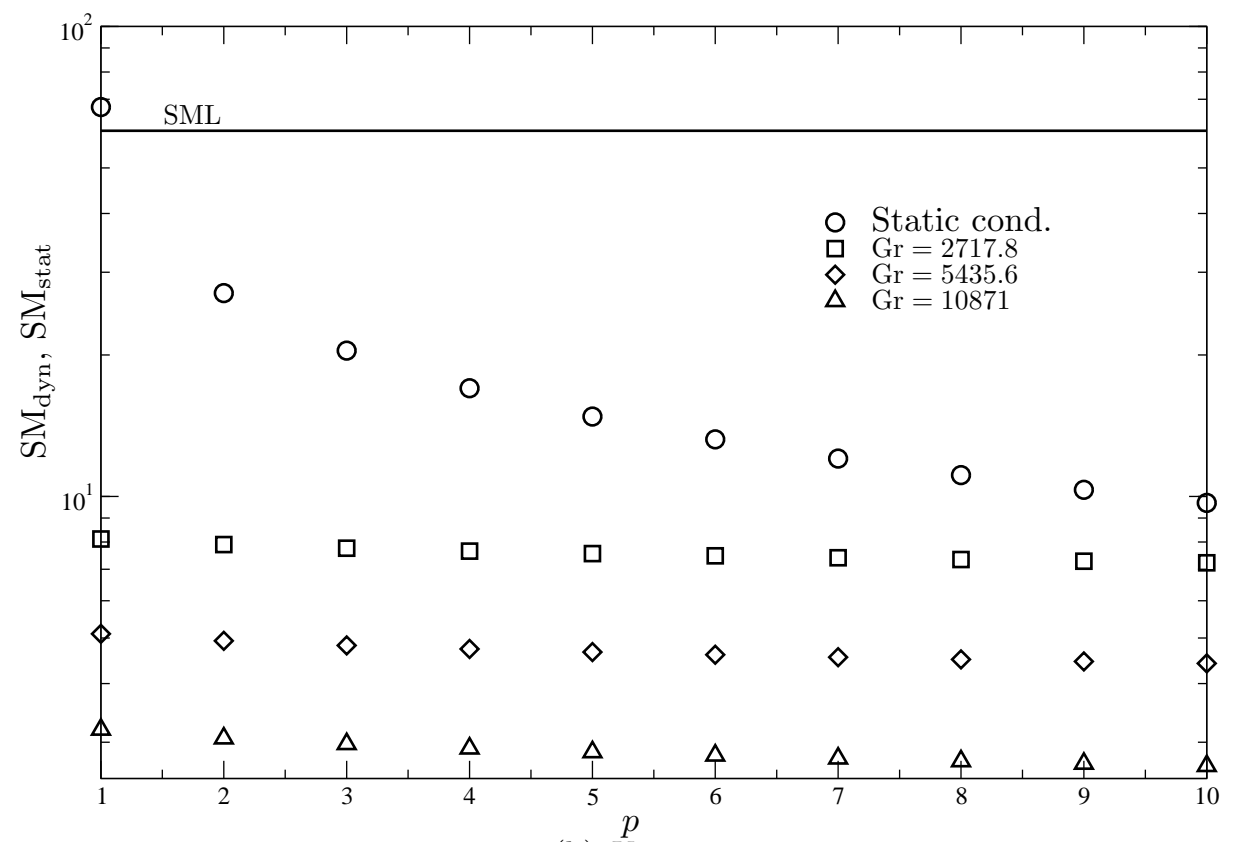

(b) $\stackrel{p}{K}=1$

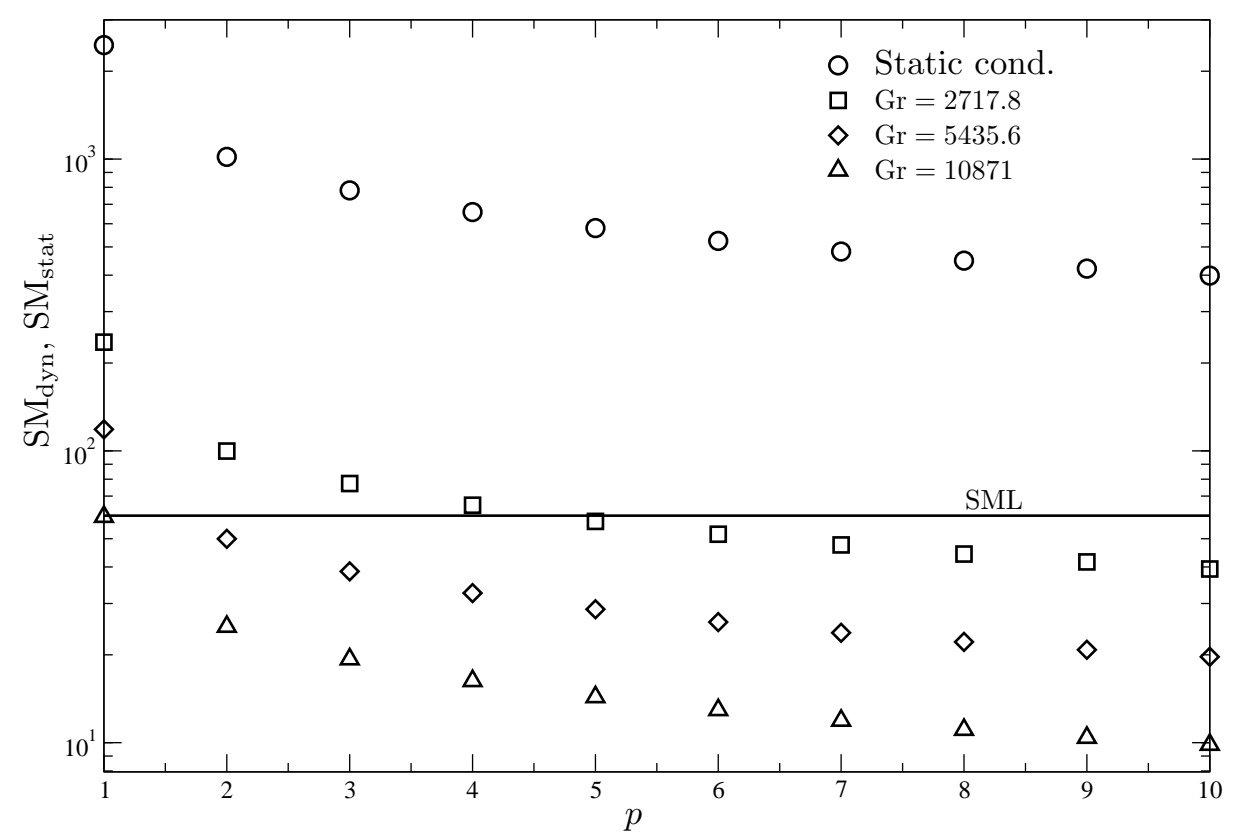

Figure 8: Specific migration of glycerol monoester plasticizer as a function of number of period $p$ with $T=10 \mathrm{~min}$ for three tube lengths and a flow rate of $1 \mathrm{l} / \mathrm{min}$ when (a) $K=10^{-3}$ and (b) $K=1$. 
The situation where the partition coefficient is equal to 1 shows that the magnitude of specific migration changes strongly between static and dynamic conditions, see Figure 8b. Around one order of magnitude is observed in the numerical data between the static and dynamic workings. While in the static condition it is impossible to be in compliance with the legislation over the ten periods simulated, the specific migration is below the SML for the longest tube $(\mathrm{Gr}=2717.8)$ after four periods. Specific migration for $\mathrm{Gr}=5435.5$ is below the SML after one period and for all periods when $\mathrm{Gr}=10871$.

These results point out the importance of the working conditions of the hoses. Due to the dilution effect and the renewing of liquid, the dynamic conditions are more appropriate and accurate to demonstrate compliance. Of course, the tube length plays on important role since the migration is a function of $L$ via the Graetz number which is inversely proportional to $L$. From the numerical results, a division by 2 of the length leads to a decrease of specific migration in the same proportion.

\section{Conclusion}

With the safety food applications in mind, a theoretical model to describe the migration of a plasticizer added in a polyvinyl chloride (PVC) material used to make hoses is developed. The aim is to compute the amount of plasticizer migrating in the liquid over a certain time. The migration of the plasticizer is a conjugated problem of transport phenomena between the diffusion of the plasticizer in solid tube to the diffusion and advection of the same substance in liquid. The final model is the coupled diffusion equation in the solid tube and the average plasticizer concentration in the liquid where the mass transfer between the two media is determined with the classical Graetz problem providing the Sherwood number.

A dedicated numerical tool to solve the conjugated problem has been developed and detailed in supplementary material of the current article. The numerical computations have been done to study the influence of the tube geometry and the flowing conditions. We point out that the average concentration of plasticizer in the liquid behaves as a function of axial coordinate with two different scalings as a function of the migration strength, $\alpha$, product of the partition coefficient (ratio of the plasticizer concentration in the liquid to this one in the solid at the PVC/liquid interface), the ratio of the PVC thickness to the interior radius and the diffusion coefficient ratio between the liquid and the solid. When $\alpha$ is smaller than one, the non-uniformity of the Sherwood number along the axial direction has a strong influence on the plasticizer concentration. Conversely, when $\alpha$ is much larger than one, the slope of the plasticizer concentration does not depend on the Sherwood number which is inversely proportional to $\alpha$ and the Graetz number. As a consequence, the plasticizer concentration is a linear function of the axial distance.

For safety issue, the amount of plasticizer migrating into the food is an important quantity to know. So, the normalized amount of plasticizer has been determined in dynamic condition and compared with the static situation. It is noteworthy that the amount of plasticizer concentration behaves as a linear function of time while in the static condition, a square root behavior is observed for small strength of migration. Conversely, when $\alpha$ is large, the amount of plasticizer concentration in the liquid scales as a square root of time but the order of magnitude is much smaller than in static condition.

The numerical computations have been used to determine the specific migration for periodic working with a relevant parameters for milk production. From the practical 
point of view, the extreme importance of the working condition is drawn. The food safety authorities requirements are fulfilled in dynamic condition for a tube dedicated to the transport of liquid with a certain flow rate. As mentioned in regulation 10/2011 [7], the conditions used to test the compliance should be the closest of the real working conditions.

These developments giving the migration in flowing conditions for the first time have to be extended in near future in threefold. First, only laminar flows have been considered although it is easy to have a Reynolds number larger than 2100 in practice. So, the model has to be extended for turbulent flow. Second, experimental works must be developed to provide values of the physical parameters such as the partition and diffusion coefficients. Finally, experimental investigations to determine specific migrations have to be undertaken.

\section{Acknowledgements}

We are indebted to Olivier Vitrac (Agro-ParisTech, INRIA, France) who learned us a lot about safety food requirements and migration processes for polymeric media. During the various enthusiastic meetings, his advice have been a huge source of information for us.

\section{Appendix A. Static conjugated problem of migration}

In this section, the static conjugated migration problem is presented. Figure A.9 sketches the two domains. The liquid is localized in the tube for a radial coordinate, $r$ lesser than $r_{i}$. The solid tube is described by the annular area such as $r \in\left[r_{i} ; r_{e}\right]$. Typical profiles of plasticizer concentrations have been sketched in the two domains where the discontinuity at the interface is mathematically defined by the balance of the concentrations weighted by the partition coefficient.

Concentrations in the solid tube and in the liquid are normalized in the same way as previously done, i.e. using equations (11). The same coordinates, $\zeta$ for $C_{p}$ and $r$ for $C_{f}$ are taken to describe the radial variation with space. The boundary conditions stay unchanged and are homogeneous over the length of the tube. Consequently, the mass concentrations in the liquid and solid domains do not depend on $z$. Finally, as it is already done for the dynamic conditions, the time is reduced by the characteristic time of diffusion in the solid tube since it is the limited phenomena.

The diffusion equations in the solid hose and in the liquid are respectively given by

$$
\begin{aligned}
\frac{\partial C_{p}}{\partial t} & =\frac{1}{1+\Delta r \zeta} \frac{\partial}{\partial \zeta}\left[(1+\Delta r \zeta) \frac{\partial C_{p}}{\partial \zeta}\right], \\
\text { Fo } \frac{\partial C_{f}}{\partial t} & =\frac{1}{r} \frac{\partial}{\partial r}\left(r \frac{\partial C_{f}}{\partial r}\right),
\end{aligned}
$$

where Fo is the ratio of the characteristic time of diffusion in the liquid, $r_{i}^{2} / D_{f}$, to the characteristic time of diffusion in the PVC, $\Delta r^{2} / D_{p}$, which can be parented to a Fourier number and written as follows

$$
\text { Fo }=\frac{D_{p}}{D_{24} \Delta r^{2}} .
$$




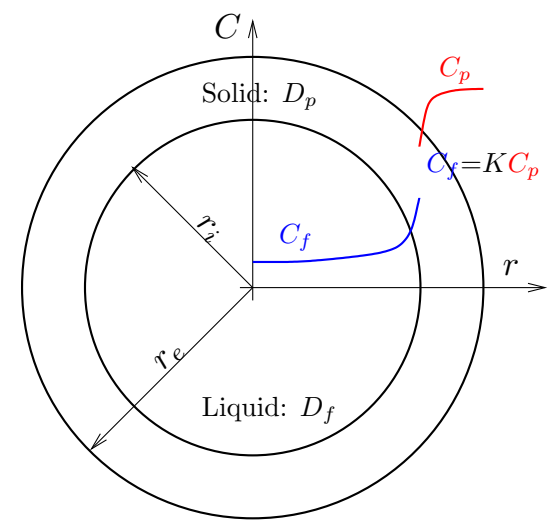

Figure A.9: Geometry and typical profiles of $C_{f}$ and $C_{p}$ in a tube of interior and exterior radii $r_{i}$ and $r_{e}$, respectively.

The initial conditions are given by

$$
\begin{gathered}
\left.C_{p}(\zeta, t)=1, \text { for } t=0, \text { and } \zeta \in\right] 0 ; 1[, \\
\left.C_{f}(r, t)=0, \text { for } t=0, \text { and } r \in\right] 0 ; 1[,
\end{gathered}
$$

and the boundary conditions by

$$
\begin{aligned}
& \frac{\partial C_{p}(\zeta, t)}{\partial \zeta}=0, \forall t>0, \text { and } \zeta=1, \\
& \left.\begin{array}{c}
C_{f}=C_{p}, \\
\frac{\partial C_{p}}{\partial \zeta}=\alpha \frac{\partial C_{f}}{\partial r} .
\end{array}\right\} \forall t>0, \text { and } \zeta=0, r=1 .
\end{aligned}
$$

The average of the plasticizer concentration in the liquid is determined by using the following integral

$$
\left\langle C_{f}\right\rangle(t)=2 \int_{0}^{1} r C_{f}(r, t) d r .
$$

This quantity which is a function of time will be used to compare to the amount of average plasticizer concentration at the exit of the tube in dynamic condition defined by (33).

A specific numerical method has been developped to solve the static problem which is detailed in supplementary material of this article.

[1] L.-Z. Zhang. Conjugate heat and mass transfer in heat mass exchanger ducts. Academic Press, 1 edition, 2013.

[2] L. Graetz. Ueber die wärmeleitungsfähigkeit von flüssigkeiten. part 1. Ann. Phys. Chem., 18:79-94, 1883.

[3] L. Graetz. Ueber die wärmeleitungsfähigkeit von flüssigkeiten. part 2. Ann. Phys. Chem., 25:337$357,1885$.

[4] R. K. Shah and A. L. London. Laminar flow forced convection in ducts. A Source book for compact heat exchanger analytical data. Academic Press, New York, 1978.

[5] R. B. Bird, W. E. Steward, and E. N. Lightfoot. Transport phenomena. John Wiley and sons, New York, 1960 
[6] E. L. Cussler. Diffusion. Mass transfer in fluid systems. Cambridge University Press, Cambridge, third edition, 2009.

[7] The European Commission. Commision regulation (EU) No 10/2011 on plastic materials and articles intended to come into contact with food. Official Journal of the European Union, L12:189, January, 14th 2011.

[8] S. Mori, M. Sakakibara, and A. Tanimoto. Steady heat transfer to laminar flow in a circular tube with conduction in tube wall. Heat Transfer Jap. Res., 3:3746-3751, 1974.

[9] M. Faghri and E. M. Sparrow. Simultaneous wall and fluid axial conduction in laminar pipe-flow heat transfer. J. Heat Transfer, 102:58-63, 1980.

[10] A. Campo and C. Schuler. Heat transfer in laminar flow through circular tubes accounting for two-dimensional wall conduction. Int. J. Heat Mass Transfer, 31(11):2251-2259, 1988.

[11] S. Bilır. Laminar flow heat transfer in pipes including two-dimensional wall and fluid axial conduction. Int. J. Heat Mass Transfer, 38(9):1619-1625, 1995.

[12] S. Bilır. Transient conjugated heat transfer in pipes involving two-dimensional wall and axial fluid conduction. Int. J. Heat Mass Transfer, 45:1781-1788, 2002.

[13] S.-X. Zhang, Y.-L. He, G. Lauriat, and W.-Q. Tao. Numerical studies of simultaneously developing laminar flow and heat transfer in microtubes with thick wall and constant outside wall temperature. Int. J. Heat Mass Transfer, 53(19-20):3977-3989, 2010.

[14] J. Crank. The mathematics of diffusion. Clarendon Press, Oxford, 1956.

[15] T. Begley, L. Castle, A. Feigenbaum, R. Franz, K. Hinrichs, T. Lickly, P. Mercea, M. Milana, A. O'brien, S. Rebre, R. Rijk, and O. Piringer. Evaluation of migration models that might be used in support of regulations for food-contact plastics. Food Addit. Contam., 22:73-90, 2005.

[16] O. Vitrac, A. Mougharbel, and A. Feigenbaum. Interfacial mass transport properties which control the migration of packaging constituents into foodstuffs. J. Food Eng., 79:1048-1064, 2007. FP939.

[17] R. Brandsch, B. Brands, R. Franz, M. Klatt, M. Milana, O. Piringer, A. Schaefer, C. Simoneau, $\mathrm{X}$. Trie, and O. Vitrac. Applicability of generally recognised diffusion models for the estimation of specific migration in support of eu directive 2002/72/ec. In C. Simoneau, editor, JRC Scientific and Technical Reports, number EUR 24514 EN 2010. Publications Office of the European Union, 2010.

[18] G. D. Smith, K. Karlsson, and U. W. Gedde. Modeling of antioxidant loss from polyolefins in hot-water applications. I: Model and application to medium density polyethylene pipes. Polym. Eng. Sci., 32:658-667, 1992.

[19] J. P. Dear and N. S. Mason. Effect of chlorine on polyethylene pipes in water distribution networks. Proc. IMechE Part L: J. Materials: Design and Applications, 220(3):97-111, 2006.

[20] G. Mittelman, J. H. Davidson, S. C. Mantell, and Y. Su. Prediction of polymer tube life for solar hot water systems: A model of antioxidant loss. Solar Energy, 82(5):452-461, 2008.

[21] Kenneth A. Mauritz, Robson F. Storey, and Scott E. George. A general free volume based theory for the diffusion of large molecules in amorphous polymers above Tg. 1. Application to di-n-alkyl phthalates in PVC. Macromolecules, 23(2):441-450, 1990.

[22] X. Fang, S. Domenek, V. Ducruet, M. Réfrégiers, and O. Vitrac. Diffusion of aromatic solutes in aliphatic polymers above glass transition temperature. Macromolecules, 46:874-888, 2013.

[23] R. Lundsgaard, G. M. Kontogeorgis, J. K. Kristiansen, and T. F. Jensen. Modeling of the migration of glycerol monoester plasticizers in highly plasticized poly(vinyl chloride). J. Vinyl Add. Tech., 15:147-158, 2009.

[24] C. R. Wilke and P. Chang. Correlation of diffusion coefficients in dilute solutions. AIChE J., 1(2):264-270, 1955.

[25] G. K. Batchelor. An introduction to fluid dynamics. Cambridge University Press, Cambridge, 1967.

[26] O. Vitrac and G. Gillet. Prediction of partition coefficients between food simulants and packaging materials using molecular simulation and a generalized flory-huggins approach. In B. Braunschweig and X. Joulia, editors, $18^{\text {th }}$ European Symposium on Computer Aided Process Engineering - ESCAPE 18. Elsevier, 2008.

[27] D. Kondepudi and I. Prigogine. Modern Thermodynamics: From Heat Engines to Dissipative Structures. John Wiley \& Sons, New York, 1998.

[28] P. J. Flory. Thermodynamics of high polymer solutions. J. Chem. Phys., 10:51-61, 1942.

[29] N. Luna, F. Méndez, and E. Mar. Transient analysis of the conjugated heat transfer in circular ducts with a power-law fluid. J. Non-Newtonian Fluid Mech., 111:69-85, 2003.

[30] G. Juncu and R. Mihail. The effect of diffusivities ratio on conjugated mass transfer from a droplet. Int. J. Heat and Mass Transfer, 30(6):1223-1226, 1987. 
[31] A. Bejan. Convection heat transfer. John Wiley \& Sons, New York, 1995.

[32] W. Guo, G. Labrosse, and R. Narayanan. The Application of the Chebyshev-Spectral Method in Transport Phenomena, volume 68 of Lecture Note in Applied and Computational Mechanics. Springer-Verlag, Berlin, 2012.

[33] E. Süli and D. F. Mayers. An Introduction to Numerical Analysis. Cambridge University Press, Cambridge (UK), 2003.

[34] The European Commission. EU directives 2002/72/EC relating to plastics materials and articles intended to come into contact with foodstuffs. Official Journal of the European Union, L220:18-55, 2002.

[35] I. S. Khattab, F. Bandarkar, M. A. A. Fakhree, and A. Jouyban. Density, viscosity, and surface tension of water+ethanol mixtures from 293 to 323 K. Korean J. Chem. Eng., 29(6):812-817, 2012. 